Department of Econometrics and Business Statistics

http://business.monash.edu/econometrics-and-business-

statistics/research/publications

\title{
A Near Unit Root Test for High- Dimensional Nonstationary Time Series
}

Bo Zhang, Jiti Gao and Guangming Pan

May 2019

Working Paper 10/19 


\title{
A Near Unit Root Test for High-Dimensional Nonstationary Time Series
}

\author{
Bo Zhang* and Jiti Gao ${ }^{\dagger}$ and Guangming Pan ${ }^{\ddagger}$
}

May 9, 2019

\begin{abstract}
This paper considers a $p$-dimensional time series model of the form

$$
\mathbf{x}_{t}-\delta_{\mathbf{t}}=\varphi\left(\mathbf{x}_{t-1}-\delta_{\mathbf{t}-\mathbf{1}}\right)+\Sigma^{1 / 2} \mathbf{y}_{t}, 1 \leq t \leq T
$$

where $\mathbf{y}_{t}=\left(Y_{t 1}, \cdots, Y_{t p}\right)^{\prime}$ and $\Sigma^{1 / 2}$ is the square root of a symmetric positive definite matrix. Here $\varphi \leq 1$ and $T(1-\varphi)$ is bounded and the linear processes $Y_{t j}$ is of the form $\sum_{k=0}^{\infty} b_{k} Z_{t-k, j}$ where $\sum_{i=0}^{\infty}\left|b_{i}\right|<\infty$ and $\left\{Z_{i j}\right\}$ are independent and identically distributed (i.i.d.) random variables with $E Z_{i j}=0, E\left|Z_{i j}\right|^{2}=1$ and $E\left|Z_{i j}\right|^{4}<\infty$. We first investigate the asymptotic behavior of the first $k$ largest eigenvalues of the sample covariance matrices of the time series model. We then propose an estimator of $\varphi$ and use it to test for near unit roots. Simulations and empirical applications are also conducted to demonstrate the performance of the statistic.
\end{abstract}

Keywords: Asymptotic normality, largest eigenvalue, linear process, near unit root test.

JEL Classification: C21, C32.

\section{Introduction}

There is a long literature about testing unit-root time series. Several testing methods have also been proposed for testing near unit root time series. The literature includes Phillips and Perron [24] for pioneering a highly popular unit-root test, Phillips [23] for establishing a novel estimation theory for a regression model associated with a unit root structure, Phillips and Xiao [25] for a survey of the relevant literature up to 1998, Phillips, Moon and Xiao

*Bo Zhang, Department of Econometrics and Business Statistics, Monash University, Caulfield East, Victoria 3145, Australia. Email: bo.zhang1@monash.edu.

${ }^{\dagger}$ Corresponding Author: Jiti Gao, Department of Econometrics and Business Statistics, Monash University, VIC 3145, Australia. Email: jiti.gao@monash.edu.

${ }^{\ddagger}$ Guangming Pan, School of Physical and Mathematical Sciences, Nanyang Technological University, Singapore, 637371. Email: gmpan@ntu.edu.sg. 
[26] for estimating autoregressive roots near unity, and Moon and Phillips [17] for GMM estimation of panel data models with roots near unity. In the past two decades or so, there have been studies on testing unit roots and/or near unit roots in panel data settings. One key contribution is by Choi [9]. Some other contributions include Levin, Lin and Chu [16], Im, Pesaran, and Shin [13], Chang [7], Pesaran [20], Pesaran, Smith and Yamagata [22] and Pesaran [21].

As demonstrated in Zhang, Pan and Gao [31], existing unit root tests available for the panel data setting are not directly applicable for the case where the cross-sectional dimension is proportional or greater than the time series sample size. To address a corresponding issue for the near unit case, we propose a new statistic for testing near unit roots in the highdimensional setting. One particular feature of the proposed test, which is quite different from those proposed in Zhang, Pan and Gao [31], is that the proposed test basically mimics the original version of the unit root test for the low-dimensional case. Since the development of the theory for the proposed test relies on some asymptotic properties of the largest eigenvalues of high-dimensional sample covariance matrices, we will briefly discuss the relevant literature in the rest of this section.

In recent years, large sample properties for high-dimensional sample covariance matrices, including their eigenvalues and eigenvectors, have been proved to be extremely useful. In fact, random matrix theory provides many useful methods for estimation and testing procedures in high-dimensional data analysis. With respect to eigenvalues and eigenvectors, asymptotic properties for the largest eigenvalues are interesting and useful. There are currently two main lines of research about asymptotic distributions of the largest eigenvalues of highdimensional random matrices. The first line of research is concerned with the Tracy-Widom law. It is well known that limiting distributions of the largest eigenvalues of high-dimensional random matrices, such as Wigner matrices, follow the Tracy-Widom law, which was originally discovered by Tracy and Widom in [28] and [29] for Gaussian Wigner ensembles. The largest eigenvalue of the Wishart matrix was investigated in Johnstone [14]. Recent progress for general sample covariance matrices has also been made, and we refer to Bao, Pan and Zhou [5], Han, Pan and Zhang[12] and El Karoui [11] among others.

Empirical data from finance, speech recognition and wireless communication often implies that some extreme eigenvalues of sample covariance matrices are well separated from the rest. This introduces the second line of research about the spiked eigenvalues, which was first proposed in Johnstone [14]. There are some significant studies in recent years on the behaviour of these spiked eigenvalues. For instance, the CLTs of the largest eigenvalues of complex Gaussian sample covariance matrices with a spiked population were investigated in Baik et al. [3], which also reported an interesting phase transition phenomenon. Baik and Silverstein [4] further considered almost sure limits of the extreme sample eigenvalues of the general spiked population. Paul [19] established a CLT for the spiked eigenvalues under the Gaussian population and the population spikes being simple. The asymptotic distribution of 
the extreme sample eigenvalues of the general spiked population with arbitrary multiplicity numbers was further reported in Bai and Yao [2].

Most of the existing studies rely on the assumption that the observations of high dimensional data are independent. However, observations of high-dimensional data in economics and finance, for example, are often highly dependent. Zhang, Pan and Gao [31] may be the first paper to deal with the largest eigenvalues of sample covariance matrices generated from high-dimensional nonstationary time series data. Unfortunately, the result of [31] is also limited. It only considers the case that there is a unit root in the time series. As we know, there are many time series with near unit roots and it is hard to use the theory of [31] to deal with them. So we need some more general results.

This paper establishes the asymptotic behaviour of the first $k$ largest eigenvalues of the sample covariance matrices of the time series model with near unit root. Another contribution of this paper is that it develops a new near unit root test. We conclude this section by giving its organization. Section 2 establishes an asymptotic distributional theory for the first several largest eigenvalues of the covariance matrix of a high-dimensional dependent time series. Section 2 also proposes a new near unit root test that is devoted to testing nonstationarity for high dimensional dependent data. Section 3 evaluates both the size and power properties of the proposed test. Section 4 concludes the paper with some remarks. Appendix A establishes some useful lemmas for the largest eigenvalues of sample covariance matrices and the proof of the main theorems in section 2. Appendix B gives and proves the truncated versions of lemmas in Appendix A by truncating linear processes. Appendix $\mathrm{C}$ gives the proofs of lemmas in Appendix A.

\section{Asymptotic Theory}

\subsection{Matrix models}

The paper is to investigate high-dimensional sample covariance matrices for nonstationary time series. Let $Y_{t j}$ be $\mathrm{MA}(q)$ with $q$ being either fixed, or tending to infinity at a certain rate, or $\infty$.

Define the linear processes $Y_{t j}$ by

$$
Y_{t j}=\sum_{k=0}^{q} b_{k} Z_{t-k, j}
$$

where $\sum_{i=0}^{q}\left|b_{i}\right|<\infty$, and $\left\{Z_{i j}\right\}$ are independent and identically distributed (i.i.d.) random variables with $E Z_{i j}=0, E\left|Z_{i j}\right|^{2}=1$ and $E\left|Z_{i j}\right|^{4}<\infty$. Suppose that $\mathbf{y}_{t}=\left(Y_{t 1}, \cdots, Y_{t p}\right)^{\prime}$ is a $p$-dimensional time series. Consider a $p$-dimensional time series model of the form:

$$
\mathbf{x}_{t}-\delta_{\mathbf{t}}=\varphi\left(\mathbf{x}_{t-1}-\delta_{\mathbf{t}-\mathbf{1}}\right)+\boldsymbol{\Sigma}^{1 / 2} \mathbf{y}_{t}, 1 \leq t \leq T
$$


where $\left\{\delta_{\mathbf{t}}\right\}_{0 \leq t \leq T}$ is non-random or independent of $\left\{\mathbf{x}_{\mathbf{t}}-\delta_{\mathbf{t}}\right\}_{0 \leq t \leq T}, \varphi \leq 1$ and $T(1-\varphi)$ is bounded.

We also define two $T \times p$ matrices $\boldsymbol{\Delta}=\left(\delta_{\mathbf{1}}, \cdots, \delta_{\mathbf{T}}\right)^{\prime}$ and $\dot{\mathbf{X}}_{\mathbf{0}}=\left(\mathbf{x}_{\mathbf{0}}-\delta_{\mathbf{0}}, \cdots, \mathbf{x}_{\mathbf{0}}-\delta_{\mathbf{0}}\right)^{\prime}$ consisting of the initial vector $\mathbf{x}_{0}$ of the time series.

Throughout the paper, we make the following assumptions about the coefficients $b_{i}$ and $\Sigma:$

(A1) $\sum_{i=0}^{q} i\left|b_{i}\right|<\infty$.

(A2) $\sum_{i=0}^{q} b_{i}=s \neq 0$.

(A3) There exist two positive constants $M_{0}$ and $M_{1}$ such that $\|\boldsymbol{\Sigma}\|_{2} \leq M_{0}$ and $\frac{\operatorname{tr}\left(\boldsymbol{\Sigma}^{\mathbf{1 / 2}}\right)}{p} \geq M_{1}$.

(A4) $T \rightarrow \infty$ and $p \rightarrow \infty$ such that $\lim _{T \rightarrow \infty} \frac{\sqrt{p}}{T}=0$.

Assumption A1 implies that the linear process can include MA( $q)$ models and AR(1) models. Assumption A2 is easily satisfied. Here $\|\cdot\|_{2}$ stands for either the spectral norm of a matrix or the Euclidean norm of a vector. Then Assumption A3 is common. Assumption A4 shows that we do not require $p$ and $T$ to be of the same order, which is being commonly used in the random matrix theory literature.

Remark 1. Assumptions A3 and A4 may lead to the main challenge of the model. Since there is no extra structural assumption on $\boldsymbol{\Sigma}$ and $p$ may be very large, it's hard to get a consistent estimator of $\boldsymbol{\Sigma}$ (or the cross-section dependence). Then most of the existing methods may not work.

We can also define $a_{i}$ as follows.

$$
a_{i}=\sum_{k=0}^{\infty} b_{k} b_{k+i}
$$

Then $a_{i}=E Y_{t j} Y_{t+i, j}$.

Now we also need to make some assumptions about $Z_{i j}$ and $\mathbf{x}_{\mathbf{0}}-\delta_{\mathbf{0}}$.

(A5) $\left\{Z_{i, j}\right\}$ are i.i.d random variables with mean zero, variance one and finite fourth moment. Let $\mathbf{z}_{t}=\left(Z_{t 1}, \cdots, Z_{t p}\right)^{\prime}$, where $t$ can be either positive or negative integer (for the purpose of introducing A7 below).

(A6) $E\left\|\mathbf{x}_{\mathbf{0}}-\delta_{0}\right\|_{2}^{2}=O(p)$.

(A7) $\mathbf{x}_{\mathbf{0}}-\delta_{\mathbf{0}}=\sum_{k=0}^{\infty} \tilde{b}_{k} \boldsymbol{\Sigma}_{\mathbf{1}}{ }^{1 / 2} \mathbf{z}_{-\mathbf{k}}+\tilde{b}_{-1} \boldsymbol{\Sigma}_{\mathbf{2}}{ }^{1 / 2} \tilde{\mathbf{z}}+\tilde{\mathbf{b}}_{-2}$, where $\left\|\boldsymbol{\Sigma}_{\mathbf{1}}\right\|_{2} \leq M_{0},\left\|\boldsymbol{\Sigma}_{\mathbf{2}}\right\|_{2} \leq M_{0}$ and $\tilde{\mathbf{z}}=$ $\left(\tilde{Z}_{1}, \cdots, \tilde{Z}_{p}\right)^{\prime}$ is independent of $\mathbf{z}_{\mathbf{t}}$ for any $t$, in which $\left\{\tilde{Z}_{j}\right\}$ are i.i.d random variables with mean zero, variance one and finite fourth moments. The coefficients satisfy $\sum_{k=0}^{\infty}\left|\tilde{b}_{k}\right|+$ $\left|\tilde{b}_{-1}\right|<\infty$ and $\left\|\tilde{\mathbf{b}}_{-2}\right\|^{2}=O(p)$. 
We would like to remark that Assumption A7 implies Assumption A6.

We next specify assumptions about $\varphi$.

(A8) The unknown $\varphi$ satisfies:

$$
\lim _{T \rightarrow \infty} T(1-\varphi)=c \geq 0
$$

(A9)

$$
\min \{q, \sqrt{p}\} \sqrt{p}(1-\varphi)=O(1)
$$

The choice of $\varphi$ in (2.4) covers many important cases where both unit-root and near-unit root scenarios can be accommodated. If Assumptions A4 and A8 hold and $q$ is bounded, Assumption A9 automatically holds.

We next specify assumptions about $\left\{\delta_{\mathbf{t}}\right\}_{1 \leq t \leq T}$.

(A10) $\left\{\delta_{\mathbf{t}}\right\}_{0 \leq t \leq T}$ is non-random or independent with $\left\{\mathbf{x}_{\mathbf{t}}-\delta_{\mathbf{t}}\right\}_{0 \leq t \leq T}$ and $\operatorname{trace}\left(\frac{1}{\sqrt{p}} \boldsymbol{\Delta}(\mathbf{I}-\mathbf{H}) \boldsymbol{\Delta}^{*}\right)=$ $\frac{1}{\sqrt{p}} \sum_{t=1}^{T} \delta_{\mathbf{t}}^{\prime}(\mathbf{I}-\mathbf{H}) \delta_{\mathbf{t}}=o_{p}\left(T^{2}\right)$.

$$
\frac{\min \{q, \sqrt{p}\}}{\sqrt{p}} \sum_{t=2}^{T}\left(\delta_{\mathbf{t}}-\delta_{\mathbf{t}-\mathbf{1}}\right)^{\prime}(\mathbf{I}-\mathbf{H})\left(\delta_{\mathbf{t}}-\delta_{\mathbf{t}-\mathbf{1}}\right)=o_{p}(T) .
$$

Remark 2. Note that the order of $\sum_{t=1}^{T} \delta_{\mathbf{t}}^{\prime}(\mathbf{I}-\mathbf{H}) \delta_{\mathbf{t}}$ may be different from that of $\sum_{t=1}^{T} \delta_{\mathbf{t}}^{\prime} \delta_{\mathbf{t}}$. For example, consider $\delta_{\mathbf{t}}=g_{t} \mathbf{1}+\tilde{\delta}_{\mathbf{t}}$ where $g_{t}$ is large and $\left\|\tilde{\delta}_{\mathbf{t}}\right\|_{2}$ is small. One may find that $\sum_{t=1}^{T} \delta_{\mathbf{t}}^{\prime} \delta_{\mathbf{t}}$ is large but $\sum_{t=1}^{T} \delta_{\mathbf{t}}^{\prime}(\mathbf{I}-\mathbf{H}) \delta_{\mathbf{t}}=\sum_{t=1}^{T} \tilde{\delta}_{\mathbf{t}}^{\prime}(\mathbf{I}-\mathbf{H}) \tilde{\delta}_{\mathbf{t}}$ is small.

\subsection{The test statistics and asymptotic theory}

We propose our statistics based on a new estimator of $\varphi$. The basic idea is as follows.

The largest challenge of our model is that $\Sigma$ is hard to be estimated. From the main theoretical results of [31], we find that when $\varphi=1$, the largest eigenvalues of $\mathbf{B}$ depend on $a_{i} \operatorname{tr}(\boldsymbol{\Sigma})$ and $a_{i} \sqrt{\operatorname{tr}\left(\boldsymbol{\Sigma}^{\mathbf{2}}\right)}$, which can be estimated. So we consider the general case of $\varphi \rightarrow 1$ and then prove that the largest eigenvalues depend on $a_{i} \operatorname{tr}(\boldsymbol{\Sigma}), a_{i} \sqrt{\operatorname{tr}\left(\boldsymbol{\Sigma}^{\mathbf{2}}\right)}$ and $\varphi$. When we obtain the estimators of $a_{i} \operatorname{tr}(\boldsymbol{\Sigma})$ and $a_{i} \sqrt{\operatorname{tr}\left(\boldsymbol{\Sigma}^{\mathbf{2}}\right)}$, we can develop a relation between the largest eigenvalue and $\varphi$. Then we can find an estimator for $\varphi$ from the largest eigenvalue.

Since there is some difficult to get an explicit expression based on $\varphi$ in some steps, we introduce $\theta$ as follows. Define $\pi>\theta_{\varphi, 1}>\cdots>\theta_{\varphi, T}>0$ are the solutions of the equation

$$
\varphi \sin T \theta+\sin (T+1) \theta=0
$$

The computational procedure is as follows.

Step 1: Define the sample covariance matrix $\mathbf{B}$ by

$$
\mathbf{B}=\frac{1}{p} \mathbf{X}(\mathbf{I}-\mathbf{H}) \mathbf{X}^{*}
$$

where $\mathbf{X}=\left(\mathbf{x}_{\mathbf{1}}, \cdots, \mathbf{x}_{\mathbf{T}}\right)^{\prime}$ and $\mathbf{H}=\frac{1}{p} \mathbf{1 1}^{\prime}$ with $\mathbf{1}$ being a $1 \times p$ column vector.

We calculate the largest eigenvalue $\rho_{1}$ of $\mathbf{B}$. 
Step 2: Estimate $q$ by calculating $\left(\mathbf{x}_{\mathbf{t}}-\mathbf{x}_{\mathbf{t}-\mathbf{1}}\right)^{\prime}(\mathbf{I}-\mathbf{H})\left(\mathbf{x}_{\mathbf{t}+\mathbf{i}}-\mathbf{x}_{\mathbf{t}+\mathbf{i}-\mathbf{1}}\right)$. Then define $m=$ $\min \{\hat{q}, \sqrt{p}\}$.

Step 3: Calculate

$$
\grave{\mu}_{m}=\sum_{t=2}^{T} \frac{\grave{\mathbf{x}}_{t, t}}{p(T-1)}+2 \sum_{j=1}^{m_{1}} \sum_{t=2}^{T-j} \frac{\grave{\mathbf{x}}_{t, t+j}}{p(T-j-1)}
$$

where

$$
\grave{\mathbf{x}}_{t, s}=\left(\mathbf{x}_{\mathbf{t}}-\mathbf{x}_{\mathbf{t}-\mathbf{1}}\right)^{\prime}(\mathbf{I}-\mathbf{H})\left(\mathbf{x}_{\mathbf{s}}-\mathbf{x}_{\mathbf{s}-\mathbf{1}}\right) .
$$

Here $\grave{\mu}_{m}$ is the estimator of the term which includes $a_{i} \operatorname{tr}(\boldsymbol{\Sigma})$.

Step 4: Define

$$
\begin{gathered}
g_{2}(\theta)=-2 \frac{\sin (T+1) \theta}{\sin T \theta}(1+\cos \theta)+\left(1+\frac{\sin (T+1) \theta}{\sin T \theta}\right)^{2}=\frac{1-\cos 2 \theta}{1-\cos 2 T \theta}, \\
\kappa(\theta)=\left(-\frac{\sin (T+1) \theta}{\sin T \theta}\right)^{m}-m \frac{\left(-\frac{\sin (T+1) \theta}{\sin T \theta}\right)^{3}-\left(-\frac{\sin (T+1) \theta}{\sin T \theta}\right)^{2 T-m}}{2(T-1)}, \\
f(\theta)=g_{2}(\theta) \kappa(\theta) .
\end{gathered}
$$

and

$$
h(\theta)=\rho_{1} f(\theta)-\grave{\mu}_{m} .
$$

We can obtain the largest solution $\hat{\theta}$ of $h(\theta)=0$ on $(0, \pi)$.

Step 5: Define

$$
g_{1}(\theta)=-\frac{\sin (T+1) \theta}{\sin T \theta} .
$$

From (2.6), we can calculate $\hat{\varphi}=g_{1}(\hat{\theta})$.

Step 6: Define

$$
\grave{S}_{\sigma^{2}, 0}=\frac{\sum_{t=2}^{[T / 2]} \sum_{s=t+[T / 2]}^{T} \grave{\mathbf{x}}_{t, s}^{2}}{\left(T-\frac{3}{2}[T / 2]\right)([T / 2]-1)}, \quad \grave{S}_{\sigma^{2}, m}=\frac{\left|\grave{\mu}_{m}\right| \sqrt{2 \frac{\grave{S}_{\sigma^{2}, 0}}{p}}}{\sum_{i=2}^{T} \frac{\grave{x}_{t, t}}{p(T-1)}} .
$$

Here $\grave{S}_{\sigma^{2}, m}$ is the estimator of the term which includes $a_{i} \sqrt{\operatorname{tr}\left(\boldsymbol{\Sigma}^{\mathbf{2}}\right)}$.

We first establish the following theorem, which will be used in the proof of Theorem 2 below.

Theorem 1. Suppose that Assumptions A1-A5 and A7-A11 hold. Then,

$$
\sqrt{p} \frac{\rho_{1} g_{2}\left(\theta_{\varphi, 1}\right) \kappa\left(\theta_{\varphi, 1}\right)-\grave{\mu}_{m}}{\grave{S}_{\sigma^{2}, m}} \rightarrow N(0,1) .
$$

Define $\widetilde{T}_{N}=\frac{\sqrt{p} \rho_{1} f^{\prime}(\hat{\theta})}{\grave{S}_{m_{2}} g_{1}^{\prime}(\hat{\theta})}(\hat{\varphi}-\varphi)$, which generalizes the test statistics $T_{N}$ of [31]. Then we can establish our main results as follows. 
Theorem 2. Suppose that Assumptions A1-A5 and A7-A11 hold.

(1) Then the solution $\hat{\theta}$ is unique such that

$$
\lim _{p, T \rightarrow \infty} P\left(\exists \hat{\theta} \in\left(\frac{T \pi}{T+1}, \frac{T \pi}{T+\frac{1}{4}}\right) \text { so that } h(\hat{\theta})=0\right)=1 \text {. }
$$

(2) As $T \rightarrow \infty$, we have

$$
\sqrt{p} \frac{\rho_{1} f^{\prime}(\hat{\theta})}{S_{\sigma^{2}, m}}\left(\hat{\theta}-\theta_{\varphi, 1}\right) \rightarrow N(0,1)
$$

(3) Moreover, we have as $T \rightarrow \infty$

$$
\widetilde{T}_{N} \rightarrow N(0,1)
$$

It is also stressed that $\widetilde{T}_{N}$ is applicable in many different choices of $\varphi$ involved in (2.4). For a general form of $\varphi=1-\frac{\chi}{T \sqrt{p}}$, we consider both unit-root and near unit root scenarios: $\chi=0, \chi=\frac{1}{\sqrt{p}}, \chi=1$ or $\chi=\sqrt{p}$. As shown in the simulation studies in Section 3 below, $\widetilde{T}_{N}$ works well numerically.

\section{Simulation}

\subsection{Estimation of $q$}

Generally, the estimation of $q$ can be done as follows. Calculate

$$
\zeta_{j}=\frac{\sum_{t=2}^{T-j} \grave{\mathbf{x}}_{t, t+j}}{p(T-j-1)}
$$

and

$$
\hat{q}=\min \left\{0 \leq i<[\sqrt{p}]:\left|\zeta_{j}\right|<p^{-1 / 4} T^{-1 / 2}, i<j<[\sqrt{p}]\right\}
$$

\subsection{Comparison with some existing tests}

There are several existing unit root tests for panel data. Some of them consider the case where there is no cross-sectional dependence and such test don't work when there is cross-sectional dependence (see, for example, the IPS test proposed in [13]). To test for nonstationarity in the panel data case with cross-sectional dependence, [7] showed that the Bootstrap method with estimation of $\boldsymbol{\Sigma}$ performs better for the case where $p$ is fixed and $T$ is large. In [31], the simulation results have showed that Bootstrap-OLS $t_{\text {ols }}^{*}$ and the F-statistic corresponding to Bootstrap-OLS $F_{\text {ols }}^{*}$ in [7] don't produce good size and power properties when $p$ is large. Now we run some simulations to show that there are similar problems for the near unit root case when $p$ is large.

We use the setting $\mathbf{y}_{\mathbf{t}}=\mathbf{z}_{\mathbf{t}}, \delta_{\mathbf{t}}=\mathbf{0}$ and $\boldsymbol{\Sigma}=\left(\Sigma_{i, j}\right)=\left(0.3^{|i-j|}\right)$. We compare the size performance of our test $T_{N}$ with the two tests $t_{\text {ols }}^{*}$ and $F_{\text {ols }}^{*}$ under $H_{0}$ with $\mathbf{x}_{\mathbf{0}}=0$. Table 1-3 
report the results of the three tests based on 1000 replications, 500 bootstrap replications and different values of $p$ and $T$. The nominal size throughout this section is set to be 0.05 .

Tables 1-3 below show that when $p$ becomes large, both $t_{o l s}^{*}$ and $F_{o l s}^{*}$ have a poor size property even though $\mathbf{y}_{\mathbf{t}}$ is independent over $t$. They don't work for the near unit root when $p$ is large.

Table 1: The empirical size of two tests under $H_{0}: \varphi=1-\frac{1}{T p}$

\begin{tabular}{crrrrrr}
\hline the test & $T \backslash p$ & 10 & 20 & 40 & 60 & 80 \\
\hline$t_{\text {ols }}^{*}$ & 40 & 0.011 & 0.007 & 0.001 & 0.000 & 0.000 \\
$F_{\text {ols }}^{*}$ & 40 & 0.025 & 0.014 & 0.001 & 0.003 & 0.001 \\
$t_{\text {ols }}^{*}$ & 60 & 0.024 & 0.016 & 0.000 & 0.000 & 0.000 \\
$F_{\text {ols }}^{*}$ & 60 & 0.042 & 0.023 & 0.000 & 0.000 & 0.002 \\
$t_{\text {ols }}^{*}$ & 80 & 0.042 & 0.020 & 0.009 & 0.001 & 0.000 \\
$F_{\text {ols }}^{*}$ & 80 & 0.050 & 0.025 & 0.012 & 0.001 & 0.000 \\
$t_{\text {ols }}^{*}$ & 160 & 0.043 & 0.029 & 0.017 & 0.010 & 0.003 \\
$F_{\text {ols }}^{*}$ & 160 & 0.066 & 0.037 & 0.020 & 0.011 & 0.003 \\
$t_{\text {ols }}^{*}$ & 320 & 0.045 & 0.032 & 0.036 & 0.024 & 0.012 \\
$F_{\text {ols }}^{*}$ & 320 & 0.061 & 0.048 & 0.047 & 0.032 & 0.015 \\
\hline
\end{tabular}

Table 2: The empirical size of two tests under $H_{0}: \varphi=1-\frac{1}{T \sqrt{p}}$

\begin{tabular}{crrrrrr}
\hline the test & $T \backslash p$ & 10 & 20 & 40 & 60 & 80 \\
\hline$t_{\text {ols }}^{*}$ & 40 & 0.021 & 0.005 & 0.000 & 0.001 & 0.000 \\
$F_{\text {ols }}^{*}$ & 40 & 0.040 & 0.007 & 0.000 & 0.001 & 0.000 \\
$t_{\text {ols }}^{*}$ & 60 & 0.026 & 0.011 & 0.002 & 0.000 & 0.000 \\
$F_{\text {ols }}^{*}$ & 60 & 0.035 & 0.017 & 0.002 & 0.000 & 0.000 \\
$t_{\text {ols }}^{*}$ & 80 & 0.042 & 0.020 & 0.002 & 0.000 & 0.000 \\
$F_{\text {ols }}^{*}$ & 80 & 0.047 & 0.026 & 0.003 & 0.000 & 0.000 \\
$t_{\text {ols }}^{*}$ & 160 & 0.040 & 0.036 & 0.021 & 0.011 & 0.001 \\
$F_{\text {ols }}^{*}$ & 160 & 0.052 & 0.046 & 0.026 & 0.032 & 0.013 \\
$t_{\text {ols }}^{*}$ & 320 & 0.058 & 0.047 & 0.027 & 0.012 & 0.001 \\
$F_{\text {ols }}^{*}$ & 320 & 0.066 & 0.058 & 0.029 & 0.037 & 0.014 \\
\hline
\end{tabular}


Table 3: The empirical size of two tests under $H_{0}: \varphi=1-\frac{1}{T}$

\begin{tabular}{crrrrrr} 
the test & $T \backslash p$ & 10 & 20 & 40 & 60 & 80 \\
\hline$t_{\text {ols }}^{*}$ & 40 & 0.021 & 0.002 & 0.000 & 0.000 & 0.000 \\
$F_{\text {ols }}^{*}$ & 40 & 0.027 & 0.0002 & 0.000 & 0.000 & 0.000 \\
$t_{\text {ols }}^{*}$ & 60 & 0.032 & 0.016 & 0.002 & 0.000 & 0.000 \\
$F_{\text {ols }}^{*}$ & 60 & 0.042 & 0.018 & 0.002 & 0.000 & 0.000 \\
$t_{\text {ols }}^{*}$ & 80 & 0.036 & 0.019 & 0.002 & 0.000 & 0.000 \\
$F_{\text {ols }}^{*}$ & 80 & 0.047 & 0.022 & 0.002 & 0.000 & 0.000 \\
$t_{\text {ols }}^{*}$ & 160 & 0.040 & 0.037 & 0.016 & 0.008 & 0.005 \\
$F_{\text {ols }}^{*}$ & 160 & 0.051 & 0.037 & 0.029 & 0.023 & 0.018 \\
$t_{\text {ols }}^{*}$ & 320 & 0.047 & 0.041 & 0.017 & 0.008 & 0.005 \\
$F_{\text {ols }}^{*}$ & 320 & 0.052 & 0.035 & 0.032 & 0.024 & 0.019 \\
\hline
\end{tabular}

\subsection{The simulation for $\widetilde{T}_{N}$}

This subsection is to conduct some simulations to investigate the size and power of $\tilde{T}_{N}$. We now consider the setting where $\mathbf{y}_{\mathbf{t}}=\psi \mathbf{z}_{\mathbf{t}-\mathbf{1}}+\mathbf{z}_{\mathbf{t}}, \psi=0.5$ and $\boldsymbol{\Sigma}=\left(\Sigma_{i, j}\right)=\left(0.3^{|i-j|}\right)$. We also set the elements of $\delta_{\mathbf{t}}$ as $\delta_{i t}=\cos \left(\frac{2 \pi(i+t)}{T}\right)$.

Since $\widetilde{T}_{N}$ works for different $\varphi$, we rewrite $\varphi=1-\frac{\chi}{T \sqrt{p}}$. The bigger $\chi$ is, the further $\varphi$ goes to 1 . The size and power results of $\widetilde{T}_{N}$ based on 1000 replications and different values of $p, T, \chi$ for the different hypothesises are reported in Tables $4-7$. The sizes and power values were all computed using asymptotic critical values from the standard normal distribution.

Tables 4-7 below provide us with the following findings.

- Table 4 shows that the test $\widetilde{T}_{N}$ has good sizes and power values even when $p$ and $T$ are as small as 10 and 20, respectively. Meanwhile, the power values increase when $\chi$ increases, and the sizes become stable even when $p$ increases to 80 . Table 4 particularly reveals some findings for the case where the null hypothesis is a unit root of the form: $H_{0}: \varphi=1$, and the alternative is a near unit root of the form: $H_{1}: \varphi=1-\frac{1}{T}$. In this setting, the power values are quite substantial with 0.239 for the case of $(p, T)=$ $(10,20)$.

- Tables 5-7 have similar features to those observed in Table 4, but there are some differences. For instances, Table 5 shows that when $H_{0}: \quad \chi=\frac{1}{\sqrt{p}}$ holds, the so-called power values under $H_{1}: \chi=0$ or $H_{1}: \chi=1$ look more like sizes. Under $H_{1}: \chi=\sqrt{p}$, however, when the departure is substantial, the power values increase significantly, and they also increase when either $p$ or $T$ increases. 
Table 4: Sizes and power values under $H_{0}: \chi=0$

\begin{tabular}{|c|c|c|c|c|c|}
\hline$p$ & $T$ & $\chi=0($ size $)$ & $\chi=\frac{1}{\sqrt{p}}$ (power) & $\chi=1$ (power) & $\chi=\sqrt{p}$ (power) \\
\hline 10 & 20 & 0.040 & 0.041 & 0.066 & 0.239 \\
\hline 10 & 30 & 0.044 & 0.044 & 0.079 & 0.308 \\
\hline 10 & 40 & 0.051 & 0.043 & 0.081 & 0.347 \\
\hline 10 & 60 & 0.053 & 0.057 & 0.094 & 0.371 \\
\hline 10 & 80 & 0.049 & 0.053 & 0.096 & 0.369 \\
\hline 20 & 20 & 0.039 & 0.031 & 0.047 & 0.408 \\
\hline 20 & 30 & 0.044 & 0.045 & 0.081 & 0.495 \\
\hline 20 & 40 & 0.053 & 0.054 & 0.090 & 0.539 \\
\hline 20 & 60 & 0.048 & 0.054 & 0.089 & 0.593 \\
\hline 20 & 80 & 0.042 & 0.054 & 0.082 & 0.606 \\
\hline 30 & 20 & 0.038 & 0.035 & 0.058 & 0.598 \\
\hline 30 & 30 & 0.049 & 0.049 & 0.064 & 0.651 \\
\hline 30 & 40 & 0.052 & 0.048 & 0.086 & 0.707 \\
\hline 30 & 60 & 0.039 & 0.044 & 0.076 & 0.731 \\
\hline 30 & 80 & 0.055 & 0.057 & 0.089 & 0.748 \\
\hline 30 & 20 & 0.043 & 0.054 & 0.093 & 0.482 \\
\hline 30 & 30 & 0.027 & 0.043 & 0.068 & 0.505 \\
\hline 30 & 40 & 0.035 & 0.040 & 0.056 & 0.523 \\
\hline 30 & 60 & 0.038 & 0.037 & 0.069 & 0.581 \\
\hline 30 & 80 & 0.034 & 0.033 & 0.059 & 0.593 \\
\hline 40 & 20 & 0.049 & 0.045 & 0.092 & 0.630 \\
\hline 40 & 30 & 0.045 & 0.036 & 0.075 & 0.666 \\
\hline 40 & 40 & 0.035 & 0.046 & 0.068 & 0.685 \\
\hline 40 & 60 & 0.026 & 0.038 & 0.072 & 0.734 \\
\hline 40 & 80 & 0.039 & 0.041 & 0.061 & 0.751 \\
\hline 60 & 20 & 0.054 & 0.061 & 0.089 & 0.801 \\
\hline 60 & 30 & 0.038 & 0.047 & 0.073 & 0.833 \\
\hline 60 & 40 & 0.040 & 0.045 & 0.060 & 0.857 \\
\hline 60 & 60 & 0.040 & 0.030 & 0.065 & 0.874 \\
\hline 60 & 80 & 0.040 & 0.036 & 0.055 & 0.913 \\
\hline 80 & 20 & 0.064 & 0.053 & 0.080 & 0.897 \\
\hline 80 & 30 & 0.032 & 0.051 & 0.066 & 0.946 \\
\hline 80 & 40 & 0.038 & 0.042 & 0.074 & 0.943 \\
\hline 80 & 60 & 0.040 & 0.044 & 0.062 & 0.953 \\
\hline 80 & 80 & 0.050 & 0.051 & 0.070 & 0.962 \\
\hline
\end{tabular}


Table 5: Sizes and power values under $H_{0}: \chi=\frac{1}{\sqrt{p}}$

\begin{tabular}{|c|c|c|c|c|c|}
\hline$p$ & $T$ & $\chi=0$ (power) & $\chi=\frac{1}{\sqrt{p}}($ size $)$ & $\chi=1$ (power) & $\chi=\sqrt{p}$ (power) \\
\hline 10 & 20 & 0.050 & 0.047 & 0.050 & 0.189 \\
\hline 10 & 30 & 0.056 & 0.057 & 0.068 & 0.280 \\
\hline 10 & 40 & 0.052 & 0.049 & 0.075 & 0.312 \\
\hline 10 & 60 & 0.039 & 0.035 & 0.071 & 0.312 \\
\hline 10 & 80 & 0.044 & 0.051 & 0.065 & 0.312 \\
\hline 20 & 20 & 0.041 & 0.033 & 0.040 & 0.400 \\
\hline 20 & 30 & 0.044 & 0.039 & 0.061 & 0.476 \\
\hline 20 & 40 & 0.045 & 0.041 & 0.064 & 0.490 \\
\hline 20 & 60 & 0.044 & 0.044 & 0.083 & 0.531 \\
\hline 20 & 80 & 0.043 & 0.044 & 0.069 & 0.562 \\
\hline 30 & 20 & 0.041 & 0.048 & 0.082 & 0.450 \\
\hline 30 & 30 & 0.027 & 0.040 & 0.060 & 0.487 \\
\hline 30 & 40 & 0.034 & 0.037 & 0.050 & 0.490 \\
\hline 30 & 60 & 0.040 & 0.036 & 0.062 & 0.550 \\
\hline 30 & 80 & 0.034 & 0.035 & 0.051 & 0.548 \\
\hline 40 & 20 & 0.046 & 0.041 & 0.079 & 0.611 \\
\hline 40 & 30 & 0.046 & 0.037 & 0.065 & 0.648 \\
\hline 40 & 40 & 0.034 & 0.040 & 0.058 & 0.669 \\
\hline 40 & 60 & 0.027 & 0.038 & 0.064 & 0.711 \\
\hline 40 & 80 & 0.035 & 0.039 & 0.054 & 0.721 \\
\hline 60 & 20 & 0.047 & 0.059 & 0.079 & 0.785 \\
\hline 60 & 30 & 0.032 & 0.044 & 0.066 & 0.824 \\
\hline 60 & 40 & 0.040 & 0.046 & 0.056 & 0.840 \\
\hline 60 & 60 & 0.037 & 0.027 & 0.061 & 0.864 \\
\hline 60 & 80 & 0.043 & 0.038 & 0.051 & 0.902 \\
\hline 80 & 20 & 0.061 & 0.046 & 0.071 & 0.894 \\
\hline 80 & 30 & 0.033 & 0.049 & 0.057 & 0.941 \\
\hline 80 & 40 & 0.036 & 0.038 & 0.069 & 0.931 \\
\hline 80 & 60 & 0.042 & 0.045 & 0.060 & 0.949 \\
\hline 80 & 80 & 0.049 & 0.049 & 0.060 & 0.958 \\
\hline
\end{tabular}


Table 6: Sizes and power values under $H_{0}: \chi=1$

\begin{tabular}{|c|c|c|c|c|c|}
\hline$p$ & $T$ & $\chi=0$ (power) & $\chi=\frac{1}{\sqrt{p}}($ power $)$ & $\chi=1($ size $)$ & $\chi=\sqrt{p}$ (power) \\
\hline 10 & 20 & 0.058 & 0.050 & 0.044 & 0.136 \\
\hline 10 & 30 & 0.062 & 0.059 & 0.052 & 0.177 \\
\hline 10 & 40 & 0.059 & 0.052 & 0.045 & 0.204 \\
\hline 10 & 60 & 0.061 & 0.057 & 0.052 & 0.200 \\
\hline 10 & 80 & 0.047 & 0.043 & 0.042 & 0.227 \\
\hline 20 & 20 & 0.064 & 0.056 & 0.035 & 0.301 \\
\hline 20 & 30 & 0.077 & 0.065 & 0.042 & 0.332 \\
\hline 20 & 40 & 0.069 & 0.066 & 0.050 & 0.381 \\
\hline 20 & 60 & 0.077 & 0.062 & 0.054 & 0.405 \\
\hline 20 & 80 & 0.071 & 0.064 & 0.048 & 0.412 \\
\hline 30 & 20 & 0.046 & 0.038 & 0.052 & 0.343 \\
\hline 30 & 30 & 0.045 & 0.031 & 0.040 & 0.350 \\
\hline 30 & 40 & 0.052 & 0.044 & 0.029 & 0.339 \\
\hline 30 & 60 & 0.067 & 0.050 & 0.042 & 0.396 \\
\hline 30 & 80 & 0.072 & 0.064 & 0.044 & 0.399 \\
\hline 40 & 20 & 0.042 & 0.039 & 0.051 & 0.459 \\
\hline 40 & 30 & 0.048 & 0.043 & 0.038 & 0.508 \\
\hline 40 & 40 & 0.045 & 0.044 & 0.033 & 0.515 \\
\hline 40 & 60 & 0.062 & 0.048 & 0.051 & 0.540 \\
\hline 40 & 80 & 0.067 & 0.064 & 0.044 & 0.544 \\
\hline 60 & 20 & 0.040 & 0.046 & 0.049 & 0.684 \\
\hline 60 & 30 & 0.050 & 0.052 & 0.046 & 0.711 \\
\hline 60 & 40 & 0.051 & 0.063 & 0.036 & 0.735 \\
\hline 60 & 60 & 0.058 & 0.056 & 0.040 & 0.781 \\
\hline 60 & 80 & 0.065 & 0.047 & 0.032 & 0.812 \\
\hline 80 & 20 & 0.057 & 0.040 & 0.047 & 0.830 \\
\hline 80 & 30 & 0.052 & 0.042 & 0.039 & 0.869 \\
\hline 80 & 40 & 0.050 & 0.049 & 0.046 & 0.879 \\
\hline 80 & 60 & 0.060 & 0.057 & 0.040 & 0.893 \\
\hline 80 & 80 & 0.066 & 0.068 & 0.034 & 0.921 \\
\hline
\end{tabular}


Table 7: Sizes and power values under $H_{0}: \chi=\sqrt{p}$

\begin{tabular}{|c|c|c|c|c|c|}
\hline$p$ & $T$ & $\chi=0$ (power) & $\chi=\frac{1}{\sqrt{p}}$ (power) & $\chi=1$ (power) & $\chi=\sqrt{p}($ size $)$ \\
\hline 10 & 20 & 0.185 & 0.159 & 0.109 & 0.054 \\
\hline 10 & 30 & 0.189 & 0.139 & 0.094 & 0.056 \\
\hline 10 & 40 & 0.202 & 0.163 & 0.114 & 0.056 \\
\hline 10 & 60 & 0.231 & 0.186 & 0.113 & 0.063 \\
\hline 10 & 80 & 0.218 & 0.171 & 0.108 & 0.053 \\
\hline 20 & 20 & 0.270 & 0.259 & 0.185 & 0.051 \\
\hline 20 & 30 & 0.343 & 0.306 & 0.204 & 0.057 \\
\hline 20 & 40 & 0.389 & 0.339 & 0.229 & 0.046 \\
\hline 20 & 60 & 0.435 & 0.391 & 0.266 & 0.057 \\
\hline 20 & 80 & 0.449 & 0.411 & 0.267 & 0.064 \\
\hline 30 & 20 & 0.502 & 0.486 & 0.374 & 0.050 \\
\hline 30 & 30 & 0.592 & 0.561 & 0.459 & 0.051 \\
\hline 30 & 40 & 0.652 & 0.656 & 0.505 & 0.041 \\
\hline 30 & 60 & 0.704 & 0.681 & 0.532 & 0.061 \\
\hline 30 & 80 & 0.721 & 0.730 & 0.533 & 0.042 \\
\hline 40 & 20 & 0.624 & 0.626 & 0.498 & 0.060 \\
\hline 40 & 30 & 0.749 & 0.742 & 0.599 & 0.046 \\
\hline 40 & 40 & 0.762 & 0.747 & 0.616 & 0.041 \\
\hline 40 & 60 & 0.825 & 0.803 & 0.666 & 0.055 \\
\hline 40 & 80 & 0.846 & 0.826 & 0.693 & 0.061 \\
\hline 60 & 20 & 0.796 & 0.795 & 0.718 & 0.046 \\
\hline 60 & 30 & 0.889 & 0.882 & 0.797 & 0.060 \\
\hline 60 & 40 & 0.908 & 0.905 & 0.820 & 0.049 \\
\hline 60 & 60 & 0.939 & 0.927 & 0.852 & 0.056 \\
\hline 60 & 80 & 0.936 & 0.948 & 0.870 & 0.043 \\
\hline 80 & 20 & 0.889 & 0.893 & 0.847 & 0.055 \\
\hline 80 & 30 & 0.957 & 0.941 & 0.917 & 0.042 \\
\hline 80 & 40 & 0.959 & 0.959 & 0.922 & 0.049 \\
\hline 80 & 60 & 0.980 & 0.973 & 0.949 & 0.061 \\
\hline 80 & 80 & 0.975 & 0.977 & 0.945 & 0.049 \\
\hline
\end{tabular}




\section{Conclusions and discussion}

This paper has proposed a new near unit test for the case where the dimensionality of a vector of time series diverges along with the sample size. The proposed test has been constructed in a similar fashion to that of the original Dickey-Fuller test, and the main difference is that the asymptotic distribution of the proposed test is standard normal, while the DF test has a nonstandard limiting distribution.

As shown in the simulation study, some of the existing tests proposed for the lowdimensional setting don't work well numerically. The proposed test however has good size and power properties in the finite-sample cases.

\section{Acknowledgements}

The authors would like to thank the Australian Research Council Discovery Grants Program for its support under Grant numbers: DP150101012 \& DP170104421.

\section{References}

[1] Bai, Z. D. and Silverstein, J. W. (2006). Spectral Analysis of Large Dimensional Random Matrices. 2nd Edition, Springer, New York.

[2] BAI, Z.D. and YAO, J.F (2008).Central limit theorems for eigenvalues in a spiked population model. Annales de l'Institut Henri Poincaré 44, 447-474.

[3] Baik, J., Ben Arous, G. and Péché, S. (2005). Phase transition of the largest eigenvalue for non-null complex sample covariance matrices. Annals of Probability 33, 1643-1697.

[4] Baik, J. and Silverstein, J. W. (2006). Eigenvalues of large sample covariance matrices of spiked population models. Journal of Multivariate Analysis 97, 1382-1408.

[5] BaO, Z. G., Pan, G. M. and Zhou, W. (2015). Universality for the largest eigenvalue of sample covariance matrices with general population. Annals of Statistics 43, 382-421.

[6] Chan, N.H. and WeI, C.Z. (1988). Limiting distributions of least squares estimates of unstable autoregressive processes. Annals of Statistics 16, 367-401.

[7] Chang, Y. (2004). Bootstrap unit root tests in panels with cross sectional dependency. Journal of Econometrics 120, 263-293.

[8] Chen, B. B. and PAN, G. M. (2012). Convergence of the largest eigenvalue of normalized sample covariance matrices when $p$ and $n$ both tend to infinity with their ratio converging to zero. Bernoulli 18, $1405-1420$.

[9] Chor, In. (2001). Unit root tests for panel data. Journal of International Money and Finance 20, 249-272.

[10] Dickey, D.A. and Fuller, W.A. (1979). Distribution of the estimators for autoregressive time series with an unit root. Journal of the American Statistical Association 74, 423-431. 
[11] El Karoui, N. (2007). Tracy-Widom limit for the largest eigenvalue of a large class of complex sample covariance matrices, Annals of Probability 35, 663-714.

[12] Han, X., Pan, G. M. and Zhang, B. (2016). The Tracy-Widom law for the largest eigenvalue of F type matrix, Annals of Statistics 44, 1564-1592.

[13] Im, K., Pesaran, M.H. and Shin, Y. (2003). Testing for unit roots in heterogeneous panels. Journal of Econometrics 115, 53-74.

[14] Johnstone, I.M. (2001). On the distribution of the largest eigenvalue in principal component analysis. Annals of Statististics 29, 295-327.

[15] Johnstone, I.M. (2007). High dimensional statistical inference and random matrices. In International Congress of Mathematicians I, 307-333.

[16] Levin, A., Lin, C.F. and Chu, C.S.J. (2002). Unit root tests in panel data: asymptotic and finitesample properties. Journal of Econometrics 108, 1-24.

[17] Moon H. R. and Phillips, P, C. B. (2004). GMM Estimation of Autoregressive Roots Near Unity with Panel Data. Econometrica 72, 467-522.

[18] Pan, G. M., GaO, J. and Yang, Y. R. (2014). Testing independence among a large number of high dimensional random vectors. Journal of the American Statistical Association 109, 600-612.

[19] Paul, D. (2007). Asymptotics of sample eigen-structure for a large dimensional spiked covariance model. Statistica Sinica 17, 1617-1642.

[20] Pesaran, M.H. (2007). A simple panel unit root test in the presence of cross-sectional dependence. Journal of Applied Econometrics 22, 265-312.

[21] Pesaran, M.H. (2015). Time Series and Panel Data Econometrics. Oxford University Press, Oxford.

[22] Pesaran, M.H., Smith, L. V. and Yamagata, T. (2013). Panel unit root tests in the presence of a multifactor error structure. Journal of Econometrics 175, 94-115.

[23] Phillips, P, C. B. (1988). Regression theory for near integrated time series. Econometrica 56, 10211044.

[24] Phillips, P, C. B. and Perron, P. (1988). Testing for a unit root in time series regression. Biometrika $75,335-346$.

[25] Phillips, P, C. B. and Xiao, Z. (1998). A Primer on unit root testing. Journal of Economic Surveys 12, $423-470$.

[26] Phillips, P, C. B., Moon H. R. and Xiao, Z. (2001). How to estimate autoregressive roots near unity? Econometric Theory 17, 26-69.

[27] Soshnikov, A. (2002). A note on universality of the distribution of the largest eigenvalues in certain sample covariance matrices. Journal of Statistical Physics 108, 1033-1056.

[28] Tracy, C. A. and Widom, H. (1994). Level-spacing distributions and the Airy kernel. Communications in Mathematical Physics 159, 151-174.

[29] Tracy, C. A. and Widom, H. (1996). On orthogonal and symplectic matrix ensembles. Communications in Mathematical Physics 177, 727-754. 
[30] Yao, J.F., Zheng, S. R. and Bai, Z.D. (2015). Large Sample Covariance Matrices and HighDimensional Data Analysis. Cambridge University Press.

[31] Zhang, B , PAn, G. M, and Gao, J. (2018). CLT for Largest eigenvalues and unit root tests for high-dimensional nonstationary time series. Annals of Statistics 46, 2186-2215.

\section{A Proofs of the main results}

We first will give some lemmas which are proved in the supplementary file.

Define for $k=1, \cdots, T$,

$$
\lambda_{\varphi, k}=\frac{1}{2 \varphi\left(1+\cos \theta_{\varphi, k}\right)+(1-\varphi)^{2}}
$$

where $\theta_{\varphi, k}$ is defined in (2.6).

We also define

$$
\gamma_{\varphi, k}=\lambda_{\varphi, k}\left(a_{0}+2 \sum_{j=1}^{\infty} a_{j}(-1)^{j} \cos \left(j \theta_{\varphi, k}\right)\right)
$$

Lemma 1. Suppose that Assumptions A1-A5 and A7-A9 hold. Let $\rho_{k}$ be the kth largest eigenvalue of $\mathbf{B}$. When $k$ is fixed, the random vector

$$
\frac{\sqrt{p}}{\sqrt{\frac{2 \operatorname{tr}\left(\boldsymbol{\Sigma}^{\mathbf{1} / \mathbf{2}}(\mathbf{I}-\mathbf{H}) \boldsymbol{\Sigma}(\mathbf{I}-\mathbf{H}) \boldsymbol{\Sigma}^{\mathbf{1} / \mathbf{2}}\right)}{p}}}\left(\frac{\rho_{1}-\gamma_{\varphi, 1} \frac{\operatorname{tr}\left(\boldsymbol{\Sigma}^{\mathbf{1} / \mathbf{2}}(\mathbf{I}-\mathbf{H}) \boldsymbol{\Sigma}^{\mathbf{1} / \mathbf{2}}\right)}{p}}{\gamma_{\varphi, 1}}, \cdots, \frac{\rho_{k}-\gamma_{\varphi, k} \frac{\operatorname{tr}\left(\boldsymbol{\Sigma}^{\mathbf{1} / \mathbf{2}}(\mathbf{I}-\mathbf{H}) \boldsymbol{\Sigma}^{\mathbf{1} / \mathbf{2}}\right)}{p}}{\gamma_{\varphi, k}}\right)^{\prime}
$$

converges weakly to a zero-mean Gaussian vector $\mathbf{w}=\left(w_{1}, \cdots, w_{k}\right)^{\prime}$ with the covariance function $\operatorname{cov}\left(w_{i}, w_{j}\right)=0$ for any $i \neq j$ and $\operatorname{var}\left(w_{i}\right)=1$.

Lemma 2. If the Assumptions A1-Ar and A9 hold, then

$$
\kappa\left(\theta_{\varphi, 1}\right)=1+O_{p}(m(1-\varphi))
$$

and

$$
\grave{\mu}_{m}=\frac{\operatorname{tr}\left(\boldsymbol{\Sigma}^{\mathbf{1} / \mathbf{2}}(\mathbf{I}-\mathbf{H}) \boldsymbol{\Sigma}^{\mathbf{1} / \mathbf{2}}\right)}{p} \kappa\left(\theta_{\varphi, 1}\right)\left(a_{0}+2 \sum_{j=1}^{\infty} a_{j}\right)+o_{p}(m(1-\varphi))+O_{p}(1-\varphi)+o_{p}\left(\frac{1}{\sqrt{p}}\right) .
$$

Lemma 3. Under the conditions specified in lemma 2,

$$
\begin{gathered}
\frac{\grave{S}_{\sigma^{2}, 0}}{p}=a_{0}^{2} \frac{\operatorname{tr}\left(\boldsymbol{\Sigma}^{\mathbf{1} / \mathbf{2}}(\mathbf{I}-\mathbf{H}) \boldsymbol{\Sigma}(\mathbf{I}-\mathbf{H}) \boldsymbol{\Sigma}^{\mathbf{1} / \mathbf{2}}\right)}{p}+o_{p}(1), \\
\grave{S}_{\sigma^{2}, m}=\frac{\left|\grave{\mu}_{m_{2}}\right| \sqrt{2 \frac{\grave{S}_{\sigma^{2}, 0}}{p}}}{\sum_{i=2}^{T} \frac{\breve{\mathbf{x}}_{i, i}}{p(T-1)}}=\left(a_{0}+2 \sum_{i=1}^{\infty} a_{i}\right) \sqrt{\frac{2 \operatorname{tr}\left(\boldsymbol{\Sigma}^{\mathbf{1} / \mathbf{2}}(\mathbf{I}-\mathbf{H}) \boldsymbol{\Sigma}(\mathbf{I}-\mathbf{H}) \boldsymbol{\Sigma}^{\mathbf{1} / \mathbf{2}}\right)}{p}}+o_{p}(1)
\end{gathered}
$$

and

$$
\frac{\gamma_{\varphi, 1} \sqrt{\frac{2 \operatorname{tr}\left(\boldsymbol{\Sigma}^{\mathbf{1 / 2}}(\mathbf{I}-\mathbf{H}) \boldsymbol{\Sigma}(\mathbf{I}-\mathbf{H}) \boldsymbol{\Sigma}^{\mathbf{1 / 2}}\right)}{p}}}{\lambda_{\varphi, 1} \grave{S}_{\sigma^{2}, m}}=1+o_{p}(1)
$$


Proof of Theorem 1: We conclude from Lemmas 1-3 that under the conditions in Theorem 1,

$$
\sqrt{p} \frac{\rho_{1}-\frac{\lambda_{\varphi, 1}}{\kappa\left(\theta_{\varphi, 1}\right)} \grave{\mu}_{m}}{\lambda_{\varphi, 1} \grave{S}_{\sigma^{2}, m}} \rightarrow N(0,1) .
$$

Note that $\lambda_{\varphi, 1}=\frac{1}{g_{2}\left(\theta_{\varphi, 1}\right)}$ and (A.4), (2.12) then follows.

Proof of Theorems 2: At first we prove (2.13). From Lemmas 1-2 we have $\frac{\rho_{1}}{\grave{\mu}_{m}}=\lambda_{\varphi, 1}\left(1+o_{p}(1)\right)$. We can define $\check{\varphi}=\varphi-\frac{1}{T}$ and $\check{\theta} \in\left(\frac{T \pi}{T+1}, \pi\right)$ such that $g_{1}(\check{\theta})=\check{\varphi}$. Then

$$
\lim _{p, T \rightarrow \infty} P(h(\check{\theta})>0)=1 .
$$

Similarly we obtain

$$
\lim _{p, T \rightarrow \infty} P\left(h\left(\frac{T \pi}{T+\frac{1}{4}}\right)<0\right)=1 .
$$

Thus (2.13) follows from the continuity of $h(\theta)$.

We next prove that the solution is unique. It suffices to prove that $f(\theta)$ is monotone on the interval $\left(\frac{T \pi}{T+1}, \frac{T \pi}{T+\frac{1}{4}}\right)$. From the chain rule $f^{\prime}(\theta)=g_{2}^{\prime}(\theta) \kappa(\theta)+g_{2}(\theta) \kappa^{\prime}(\theta)$. It is straightforward to verify that $\kappa(\theta)=1+o_{p}(1), \kappa^{\prime}(\theta)=O_{p}\left(m_{1}\right)$ and $g_{2}(\theta)=O_{p}\left(\frac{1}{T^{2}}\right)$. As for $g_{2}^{\prime}(\theta)$ we have

$$
g_{2}^{\prime}(\theta)=\frac{\sin 2 \theta \sin T \theta-2 T \cos T \theta \sin ^{2} \theta}{\sin ^{3} T \theta} .
$$

We rewrite $\theta=\frac{T \pi}{T+\imath}$ where $0<\imath<1$. Then

$$
\begin{gathered}
g_{1}(\theta)=-\frac{\sin (T+1) \theta}{\sin T \theta}=\frac{\sin \frac{T(1-\imath) \pi}{T+\imath}}{\sin \frac{T \imath \pi}{T+\imath}} . \\
g_{2}^{\prime}(\theta)=\frac{\sin \frac{2 T \pi}{T+\imath} \sin \left(T \pi-\frac{T \imath \pi}{T+\imath}\right)-2 T \cos \left(T \pi-\frac{T \imath \pi}{T+\imath}\right) \sin ^{2} \frac{T \pi}{T+\imath}}{\sin ^{3}\left(T \pi-\frac{T \imath \pi}{T+\imath}\right)} \\
=2 \sin \frac{T \pi}{T+\imath} \frac{\cos \frac{T \pi}{T+\imath} \sin \left(T \pi-\frac{T \imath \pi}{T+\imath}\right)-T \cos \left(T \pi-\frac{T \imath \pi}{T+\imath}\right) \sin \frac{T \pi}{T+\imath}}{\sin ^{3}\left(T \pi-\frac{T \imath \pi}{T+\imath}\right)} \\
=2 \sin \frac{T \pi}{T+\imath} \frac{\cos \frac{T \pi}{T+\imath}(-1)^{T+1} \sin ^{\frac{T \imath \pi}{T+\imath}}+T(-1)^{T+1} \cos \frac{T \imath \pi}{T+\imath} \sin \frac{T \pi}{T+\imath}}{(-1)^{T+1} \sin ^{3} \frac{T \imath \pi}{T+\imath}} \\
=2 \sin \frac{\imath \pi}{T+\imath} \frac{-\cos \frac{\imath \pi}{T+\imath} \sin \frac{T \imath \pi}{T+\imath}+T \cos \frac{T \imath \pi}{T+\imath} \sin \frac{\imath \pi}{T+\imath}}{\sin ^{3} \frac{T \imath \pi}{T+\imath}} .
\end{gathered}
$$

When $\frac{1}{4}<\imath<1,-\cos \frac{\imath \pi}{T+\imath} \sin \frac{T \imath \pi}{T+\imath}+T \cos \frac{T \imath \pi}{T+\imath} \sin \frac{\imath \pi}{T+\imath}<0$ has a constant order. It follows that there is a constant $\jmath>0$ such that

$$
g_{2}^{\prime}(\theta) T<-\jmath
$$

when $\frac{1}{4}<\imath<1$. This, together with $\kappa(\theta)=1+o_{p}(1), \kappa^{\prime}(\theta)=O_{p}\left(m_{1}\right)$ and $g_{2}(\theta)=O_{p}\left(\frac{1}{T^{2}}\right)$, implies that there is a constant $\jmath_{1}>0$ such that

$$
T f^{\prime}(\theta)<-\jmath_{1}
$$

Then the solution is unique. 
Now we prove (2.14). Since $\hat{\theta} \in\left(\frac{T \pi}{T+1}, \pi\right)$ is the solution of $h(\theta)=0$, we can rewrite (2.12) as

$$
\sqrt{p} \frac{\rho_{1} f\left(\theta_{\varphi, 1}\right)-\rho_{1} f(\hat{\theta})}{\grave{S}_{\sigma^{2}, m_{2}}} \rightarrow N(0,1) .
$$

This leads to $f\left(\theta_{\varphi, 1}\right)-f(\hat{\theta})=O_{p}\left(\frac{1}{T^{2} \sqrt{p}}\right)$. This, together with (A.14), implies that

$$
\hat{\theta}-\theta_{\varphi, 1}=O_{p}\left(\frac{1}{T \sqrt{p}}\right) .
$$

Then we use Taylor expansions. We only need to prove $f^{\prime \prime}(\tilde{\theta})=O_{p}(1)$ for any $\tilde{\theta}$ between $\theta_{\varphi, 1}$ and $\hat{\theta}$. Since (A.16) ensures that $\theta_{\varphi, 1}$ and $\hat{\theta}$ are very close, it's not hard. The proof of (2.15) is similar. We ignore the details.

\section{B Results for truncated matrices}

This section is to consider the truncated version of the sample covariance matrix. The overall strategy of the proof, which needs more delicate analysis, is similar to that of [31]. However for the completeness of the proof and for facilitating the readers we repeat some necessary steps in [31] and still use the same notation as in [31].

To this end, define

$$
Y_{i j, l}=\sum_{k=0}^{l} b_{k} Z_{i-k, j}
$$

with $l=\max \{p, T\}$, a truncated version of $Y_{t j}$ in (2.1). However, to simplify notation, we let $b_{i}=0$ for all $i>l$ in this section, so that we still use $Y_{i j}$ instead of $Y_{i j, l}$. In this way $a_{i}$ defined in (2.3) and $Y_{t j}$ in (2.1) respectively become

$$
a_{i}=\sum_{k=0}^{l-i} b_{k} b_{k+i}, \quad Y_{t j}=\sum_{k=0}^{l} b_{k} Z_{t-k, j} .
$$

Furthermore let $\mathbf{F}=\left(F_{i j}\right)$ be a $T \times(T+l)$ matrix with

$$
F_{i j}= \begin{cases}b_{l+i-j} & i \leq j \leq i+l \\ 0 & \text { otherwise }\end{cases}
$$

It follows that $\mathbf{Y}=\mathbf{F} \mathbf{Z}_{\mathbf{p}}$, where $\mathbf{Z}_{\mathbf{p}}$ is a $(T+l) \times p$ random matrix with $\left(\mathbf{Z}_{\mathbf{p}}\right)_{i, j}=Z_{i-l, j}$. For the sake of notation simplicity, we below denote $\mathbf{Z}_{\mathbf{p}}$ by $\mathbf{Z}$ and $\left(\mathbf{Z}_{\mathbf{p}}\right)_{i, j}$ by $Z_{i j}$. Let $\mathbf{A}=\left(A_{i j}\right)_{T \times T}=\left(a_{|i-j|}\right)_{T \times T}$. We then have $\mathbf{A}=\mathbf{F} \mathbf{F}^{\prime}$. We would remind readers that $l$ depends on $T$ so that $a_{|i-j|}$ depends on $\mathrm{T}$.

We also assume that $\mathbf{x}_{\mathbf{0}}=\mathbf{0}$ and $\delta_{\mathbf{t}}=\mathbf{0}$ in this section.

Note that we have studied the case $\varphi=1$ in [31]. Below we deal with with the case of $\varphi<1$.

\section{B.1 Eigenvalues of $\mathrm{C}_{\varphi} \mathrm{AC}_{\varphi}{ }^{*}$}

Write

$\mathbf{B}=(1 / p) \mathbf{X}(\mathbf{I}-\mathbf{H}) \mathbf{X}^{*}=(1 / p) \mathbf{C}_{\varphi} \mathbf{Y} \boldsymbol{\Sigma}^{\mathbf{1} / \mathbf{2}}(\mathbf{I}-\mathbf{H}) \boldsymbol{\Sigma}^{\mathbf{1} / \mathbf{2}} \mathbf{Y}^{*} \mathbf{C}_{\varphi}{ }^{*}=(1 / p) \mathbf{C}_{\varphi} \mathbf{F} \mathbf{Z}_{\mathbf{p}} \boldsymbol{\Sigma}^{\mathbf{1} / \mathbf{2}}(\mathbf{I}-\mathbf{H}) \boldsymbol{\Sigma}^{\mathbf{1} / \mathbf{2}} \mathbf{Z}_{\mathbf{p}}^{*} \mathbf{F}^{*} \mathbf{C}_{\varphi}{ }^{*}$ 
We below investigate the eigenvalues and eigenvectors of $\mathbf{C}_{\varphi} \mathbf{F} \mathbf{F}^{*} \mathbf{C}_{\varphi}{ }^{*}=\mathbf{C}_{\varphi} \mathbf{A C}_{\varphi}{ }^{*}$ at first. These are crucial steps.

Since it's very hard to find the eigenvalues of $\mathbf{C}_{\varphi} \mathbf{A} \mathbf{C}_{\varphi}{ }^{*}$ directly we use the following strategy. At first, we note that the eigenvalues of $\mathbf{C}_{\varphi} \mathbf{A} \mathbf{C}_{\varphi}{ }^{*}$ and $\mathbf{A C}_{\varphi}^{*} \mathbf{C}_{\varphi}$ are the same. We obtain the eigenvalues and eigenvectors of $\mathbf{C}_{\varphi}^{*} \mathbf{C}_{\varphi}$ by first studying $\left(\mathbf{C}_{\varphi}^{*} \mathbf{C}_{\varphi}\right)^{-1}$. The next key step is to approximate the eigenvalues of $\mathbf{C A C} *$. Our results are summarized in the following series of Lemmas and Theorems.

The first two lemmas describe the eigenvalues of $\mathbf{C}_{\varphi}^{*} \mathbf{C}_{\varphi}$ and decide their limits.

Lemma 4. Let $\lambda_{\varphi, 1} \geq \lambda_{\varphi, 2} \geq \cdots \geq \lambda_{\varphi, T} \geq 0$ be the eigenvalues of $\mathbf{C}_{\varphi}^{*} \mathbf{C}_{\varphi}$. We then have

$$
\lambda_{\varphi, k}=\frac{1}{2 \varphi\left(1+\cos \theta_{\varphi, k}\right)+(1-\varphi)^{2}} \text { with } \pi>\theta_{\varphi, 1}>\cdots>\theta_{\varphi, T}>0,
$$

where $\pi>\theta_{\varphi, 1}>\cdots>\theta_{\varphi, T}>0$ are the solutions of the equation

$$
\varphi \sin T \theta+\sin (T+1) \theta=0 .
$$

Lemma 5. (Lemma C.2 in [31]) Using the notation in Lemma 4,

$$
\lim _{T \rightarrow \infty} \frac{\lambda_{1, k}}{T^{2}}=\frac{4}{\pi^{2}(2 k-1)^{2}}
$$

for any fixed $k$.

Lemma 6. Using the notation in Lemma 4, under the assumption (A9),

$$
\lim _{T \rightarrow \infty} \frac{\lambda_{\varphi, k}}{T^{2}}>0
$$

for any fixed $k$. Futhermore, when $T$ is big enough there exists a independent constant $\Upsilon>0$ such that

$$
\lim _{T \rightarrow \infty} \frac{\lambda_{1, k}-\lambda_{\varphi, k}}{\lambda_{1, k}} \leq \Upsilon T(1-\varphi)
$$

for any $k$.

Lemma 7 below specifies the eigenvectors of $\mathbf{C}_{\varphi}^{*} \mathbf{C}_{\varphi}$.

Lemma 7. Let $\tilde{\mathbf{x}}_{\mathbf{k}}=\left(x_{k, 1}, \cdots, x_{k, T}\right)^{\prime}$ be a $T \times 1$ vector with

$$
x_{k, i}=(-1)^{T-i} \sin (T-i+1) \theta_{\varphi, k}, \quad-l \leq i \leq T+l .
$$

Then $\left\{\tilde{\mathbf{x}}_{\mathbf{k}}, 1 \leq k \leq T\right\}$ are orthogonal and satisfy for any $k$

$$
\mathbf{C}_{\varphi}^{*} \mathbf{C}_{\varphi} \tilde{\mathbf{x}}_{\mathbf{k}}=\lambda_{\varphi, k} \tilde{\mathbf{x}}_{\mathbf{k}}
$$

Lemma 8.

$$
\Sigma_{j=1}^{T}\left(x_{k, j}\right)^{2}=\frac{T}{2}+o(T) .
$$

Let

$$
\tilde{\mathbf{y}}_{\mathbf{k}}=\frac{\tilde{\mathbf{x}}_{\mathbf{k}}}{\left\|\tilde{\mathbf{x}}_{\mathbf{k}}\right\|}
$$

Then $\left\{\tilde{\mathbf{y}}_{\mathbf{k}}\right\}_{1 \leq k \leq T}$ are orthogonal and the $j$ th element of $\tilde{\mathbf{y}}_{\mathbf{k}}, y_{k, j}$, satisfies

$$
\left|y_{k, j}\right|=\frac{\left|x_{k, j}\right|}{\left\|\tilde{\mathbf{x}}_{\mathbf{k}}\right\|}=O\left(\frac{1}{\sqrt{T}}\right) .
$$


Lemma 9 gives the approximation to the eigenvalues of $\mathbf{A C}_{\varphi}^{*} \mathbf{C}_{\varphi}$.

Lemma 9. Define $\gamma_{\varphi, k}$ by

$$
\gamma_{\varphi, k}=\lambda_{\varphi, k}\left(a_{0}+2 \sum_{1 \leq j \leq T-1} a_{j}(-1)^{j} \cos \left(j \theta_{\varphi, k}\right)\right) .
$$

When $T(1-\varphi)$ is bounded, for any fixed constant $k \geq 1$, there is a constant $c_{\varphi, k}$ such that

$$
\lim _{T \rightarrow \infty} \frac{\gamma_{\varphi, k}}{T^{2}}=c_{\varphi, k}>0 .
$$

Let $\beta_{\varphi, 1} \geq \beta_{\varphi, 2} \geq \cdots \geq \beta_{\varphi, T}$ be the eigenvalues of $\mathbf{A C}_{\varphi}^{*} \mathbf{C}_{\varphi}$. If $\mathbf{A}$ satisfies the assumptions (A1) and (A2), then for any fixed integers $i \geq 1$ and $j \geq 1$ the following holds

$$
\left|\frac{\beta_{\varphi, i}-\gamma_{\varphi, i}}{\gamma_{\varphi, j}}\right|=O\left(T^{-1}\right) .
$$

For any $\epsilon>0$ there exists $T_{0}$ and $k_{0}$ where $k_{0}$ is a fixed number independent of $T$ such that when $T \geq T_{0}$ and $k \geq k_{0}$,

$$
\left|\frac{\beta_{\varphi, k}}{\gamma_{\varphi, 1}}\right| \leq \epsilon
$$

Lemma 10. (Lemma C.5 in [31]) Suppose that A satisfies the assumptions (A1) and (A2). Then

$$
\operatorname{tr}\left(\mathbf{A C}_{\mathbf{1}}^{*} \mathbf{C}_{\mathbf{1}}\right)=a_{0} \frac{(T+1) T}{2}+\sum_{1 \leq j \leq T-1} a_{j}(T-j+1)(T-j)
$$

and

$$
\lim _{T \rightarrow \infty} \frac{\beta_{1, k}}{\operatorname{tr}\left(\mathbf{A} \mathbf{C}_{\mathbf{1}}^{*} \mathbf{C}_{\mathbf{1}}\right)}=\lim _{T \rightarrow \infty} \frac{\gamma_{1, k}}{\operatorname{tr}\left(\mathbf{A} \mathbf{C}_{\mathbf{1}}^{*} \mathbf{C}_{\mathbf{1}}\right)}=\frac{8}{\pi^{2}(2 k-1)^{2}} .
$$

Lemma 11. (Lemma C.6 in [31]) Suppose that A satisfies the assumptions (A1) and (A2). For any $\epsilon>0$, we can find $T_{0}$ and $k_{0}$, where $k_{0}$ is a finite number independent of $T$, such that when $T \geq T_{0}$,

$$
\left|\frac{\sum_{k>k_{0}} \beta_{1, k}}{\gamma_{1,1}}\right|<\epsilon
$$

Lemma 12. Suppose that $\mathbf{A}$ satisfies the assumptions (A1) and (A2). For any $\epsilon>0$, we can find $T_{0}$ and $k_{0}$, where $k_{0}$ is a finite number independent of $T$, such that when $T \geq T_{0}$,

$$
\left|\frac{\sum_{k>k_{0}} \beta_{\varphi, k}}{\gamma_{\varphi, 1}}\right|<\epsilon
$$

Proof of Lemma 4:

Let $\mathbf{M}_{\mathbf{T}}=\left(\mathbf{C}_{\varphi}^{*} \mathbf{C}_{\varphi}\right)^{-1}$. Define the characteristic function of $\mathbf{M}_{\mathbf{T}}$ by $g_{T}(\lambda)=\operatorname{det}\left(\lambda \mathbf{I}_{\mathbf{T}}-\mathbf{M}_{\mathbf{T}}\right)$. We can verify that the entries of the inverse matrix $\mathbf{C}_{\varphi}{ }^{-1}$, a $T \times T$ lower triangular matrix, are of the form

$$
C_{\varphi, i j}^{-1}= \begin{cases}1 & i=j, \\ -\varphi & i=j+1, \\ 0 & \text { otherwise. }\end{cases}
$$

It follows that $M_{i, j}$, the elements of $\mathbf{M}_{\mathbf{T}}=\left(\mathbf{C}_{\varphi}^{*} \mathbf{C}_{\varphi}\right)^{-1}$, satisfy

$$
M_{i j}= \begin{cases}1 & i=j=1 \\ 1+\varphi^{2} & i=j>1 \\ -\varphi & |i-j|=1 \\ 0 & \text { otherwise }\end{cases}
$$


By the cofactor expansion we obtain a recurrence relation as following

$$
g_{T}(\lambda)=\left(\lambda-1-\varphi^{2}\right) g_{T-1}(\lambda)-\varphi^{2} g_{T-2}(\lambda)
$$

Consider $\lambda \in(0,4)$ at first. Hence we may write $\lambda=\lambda(\theta)=1+\varphi^{2}+2 \varphi \cos \theta$. We can further solve (B.18) to get

$$
g_{T}(\lambda)=\frac{\varphi^{T+1} \sin T \theta+\varphi^{T} \sin (T+1) \theta}{\sin \theta} .
$$

When $\sin \theta \neq 0, g_{T}(\lambda)=0$ is equivalent to

$$
\varphi \sin T \theta+\sin (T+1) \theta=0 .
$$

Let $h_{T}(\theta)=\varphi \sin T \theta+\sin (T+1) \theta$. For any $1 \leq k \leq T$,

$$
h_{T}\left(\frac{k \pi}{T+1 / 2}\right)=\varphi \sin \frac{k T \pi}{T+1 / 2}+\sin \frac{k(T+1) \pi}{T+1 / 2}=(-1)^{k}(1-\varphi) \sin \frac{2 k \pi}{2 T+1}
$$

and

$$
h_{T}\left(\frac{k \pi}{T+1}\right)=\varphi \sin \frac{k T \pi}{T+1}=\varphi(-1)^{k-1} \sin \frac{(T+1-k) \pi}{T+1} .
$$

Then $\exists \theta_{\varphi, T+1-k} \in\left[\frac{k \pi}{T+1}, \frac{k \pi}{T+1 / 2}\right]$ such that $h_{T}\left(\theta_{\varphi, k}\right)=0$. So (B.3) gives $T$ different solutions which satisfy $h_{T}(\theta)=0$ and $\sin \theta \neq 0$. On the other hand, observe that there are at most $\mathrm{T}$ solutions for $g_{T}(\lambda)=0$. The proof of (B.3) is complete.

Proof of Lemma 6: Recalling the proof of Lemma 4, $\theta_{\varphi, k} \in\left[\frac{(T+1-k) \pi}{T+1}, \frac{(T+1-k) \pi}{T+1 / 2}\right)$. Then we can find that both $T^{2}\left(1+\cos \theta_{\varphi, k}\right)$ and $T^{2}(1-\varphi)^{2}$ are bounded for any fixed $k$. So we can proof (B.6).

We next prove (B.7), Write

$\lambda_{1, k}-\lambda_{\varphi, k}=\frac{1}{2+2 \cos \theta_{1, k}}-\frac{1}{1+\varphi^{2}+2 \varphi \cos \theta_{\varphi, k}}=\frac{(1-\varphi)^{2}+2(\varphi-1)\left(1+\cos \theta_{\varphi, k}\right)+2\left(\cos \theta_{\varphi, k}-\cos \theta_{1, k}\right)}{\left(2+2 \cos \theta_{1, k}\right)\left(1+\varphi^{2}+2 \varphi \cos \theta_{\varphi, k}\right)}$.

For any $k$, we define

$$
\varpi_{k}=\theta_{1, k}-\theta_{\varphi, k}=\frac{(T+1-k) \pi}{T+1 / 2}-\theta_{\varphi, k}
$$

Then $0<\varpi_{T-k+1} \leq \frac{k \pi}{T(2 T+1)}$,

$$
\frac{\lambda_{1, k}-\lambda_{\varphi, k}}{\lambda_{1, k}}=\frac{(1-\varphi)^{2}+2(\varphi-1)\left(1+\cos \theta_{\varphi, k}\right)+4 \sin \left(\theta_{1, k}-\frac{\varpi_{k}}{2}\right) \sin \frac{\varpi_{k}}{2}}{1+\varphi^{2}+2 \varphi \cos \theta_{\varphi, k}} .
$$

Note that

$$
0<\frac{(1-\varphi)^{2}}{1+\varphi^{2}+2 \varphi \cos \theta_{\varphi, k}} \leq \frac{\lambda_{1,1}}{T^{2}} T^{2}(1-\varphi)^{2}
$$

and

$$
\left|\frac{(\varphi-1)\left(1+\cos \theta_{\varphi, k}\right)}{1+\varphi^{2}+2 \varphi \cos \theta_{\varphi, k}}\right|=(1-\varphi)\left|\frac{\left(1+\cos \theta_{\varphi, k}\right)}{(1-\varphi)^{2}+2 \varphi\left(1+\cos \theta_{\varphi, k}\right)}\right| \leq \frac{1-\varphi}{2 \varphi} .
$$

From (B.4), (B.19) and (B.20), we can find that

$$
(1-\varphi) \sin T \theta_{\varphi, k}=\sin T \theta_{\varphi, k}+\sin (T+1) \theta_{\varphi, k}=2 \sin (T+1 / 2) \theta_{\varphi, k} \cos \frac{\theta_{\varphi, k}}{2} .
$$

It follows that

$$
(1-\varphi) \sin T\left(\frac{(T+1-k) \pi}{T+1 / 2}-\varpi_{k}\right)=2 \sin (T+1 / 2)\left(\frac{(T+1-k) \pi}{T+1 / 2}-\varpi_{k}\right) \cos \frac{\theta_{\varphi, k}}{2} .
$$


Then

$$
\begin{gathered}
(1-\varphi) \sin \left((T+1-k) \pi-\frac{T+1-k}{2 T+1} \pi-T \varpi_{k}\right)=2 \sin \left((T+1-k) \pi-(T+1 / 2) \varpi_{k}\right) \cos \frac{\theta_{\varphi, k}}{2} . \\
(1-\varphi) \sin \left(\frac{T+1-k}{2 T+1} \pi+T \varpi_{k}\right)=2 \sin (T+1 / 2) \varpi_{k} \cos \frac{\theta_{\varphi, k}}{2} .
\end{gathered}
$$

Then we can find that

$$
(1-\varphi) \sin \left(\frac{T+1-k}{2 T+1} \pi\right) \cos T \varpi_{k}+(1-\varphi) \sin T \varpi_{k} \cos \left(\frac{T+1-k}{2 T+1} \pi\right)=2 \sin (T+1 / 2) \varpi_{k} \cos \frac{\theta_{\varphi, k}}{2} .
$$

Recall that $0<\varpi_{T-k+1} \leq \frac{k \pi}{T(2 T+1)}$ and $\theta_{\varphi, k} \in\left[\frac{(T+1-k) \pi}{T+1}, \frac{(T+1-k) \pi}{T+1 / 2}\right]$. Then $\sin (T+1 / 2) \varpi_{k} \cos \frac{\theta_{\varphi, k}}{2} \neq$ 0 . It follows that

$$
(1-\varphi) \frac{\sin \frac{T+1-k}{2 T+1} \pi \cos T \varpi_{k}}{2 \sin (T+1 / 2) \varpi_{k} \cos \frac{\theta_{\varphi, k}}{2}}+(1-\varphi) \frac{\sin T \varpi_{k} \cos \frac{T+1-k}{2 T+1} \pi}{2 \sin (T+1 / 2) \varpi_{k} \cos \frac{\theta_{\varphi, k}}{2}}=1 .
$$

Note that $0<T \varpi_{k}<(T+1 / 2) \varpi_{k} \leq \frac{T+1-k \pi}{2 T} \leq \frac{\pi}{2}$, and

$$
0<\frac{\sin T \varpi_{k}}{\sin (T+1 / 2) \varpi_{k}}<1
$$

Similarly, since $\theta_{\varphi, k} \in\left[\frac{(T+1-k) \pi}{T+1}, \frac{(T+1-k) \pi}{T+1 / 2}\right)$, we have

$$
0<\frac{\cos \frac{T+1-k}{2 T+1} \pi}{\cos \frac{(T+1-k) \pi}{2 T+2}}<\frac{\cos \frac{T+1-k}{2 T+1} \pi}{\cos \frac{\theta_{\varphi, k}}{2}}<\frac{\cos \frac{T+1-k}{2 T+1} \pi}{\cos \frac{(T+1-k) \pi}{2 T+1}}=1 .
$$

(B.26)-(B.27) imply that

$$
(1-\varphi) \frac{\sin T \varpi_{k} \cos \frac{T+1-k}{2 T+1} \pi}{2 \sin (T+1 / 2) \varpi_{k} \cos \frac{\theta_{\varphi, k}}{2}}=O(1-\varphi) .
$$

This, together with (B.1), implies that

$$
(1-\varphi) \frac{\sin \frac{T+1-k}{2 T+1} \pi \cos T \varpi_{k}}{2 \sin (T+1 / 2) \varpi_{k} \cos \frac{\theta_{\varphi, k}}{2}}=1+O(1-\varphi) .
$$

Then

$$
\tan T \varpi_{k} \cos \frac{\varpi_{k}}{2}+\sin \frac{\varpi_{k}}{2}=\frac{\sin (T+1 / 2) \varpi_{k}}{\cos T \varpi_{k}}=(1-\varphi) \frac{\sin \frac{T+1-k}{2 T+1} \pi}{2 \cos \frac{\theta_{\varphi, k}}{2}}(1+O(1-\varphi)) .
$$

Since $0<\varpi_{k} \leq \frac{(T-k+1) \pi}{T(2 T+1)}<\frac{\pi}{2 T}$,

$$
\cos \frac{\varpi_{k}}{2}=1+O\left(\frac{(T-k+1)^{2}}{T^{4}}\right)
$$

and

$$
\sin \frac{\varpi_{k}}{2}=O\left(\frac{\tan T \varpi_{k}}{T}\right) .
$$

(B.28)-(B.30) imply that

$$
\tan T \varpi_{k}\left(1+O\left(\frac{1}{T}\right)\right)=(1-\varphi) \frac{\sin \frac{T+1-k}{2 T+1} \pi}{2 \cos \frac{\theta_{\varphi, k}}{2}}(1+O(1-\varphi)) .
$$


Since $T(1-\varphi)$ is finite, we have

$$
\tan T \varpi_{k}=(1-\varphi) \frac{\sin \frac{T+1-k}{2 T+1} \pi}{2 \cos \frac{\theta_{\varphi, k}}{2}}\left(1+O\left(\frac{1}{T}\right)\right) .
$$

Similarly, since $\theta_{\varphi, k} \in\left[\frac{(T+1-k) \pi}{T+1}, \frac{(T+1-k) \pi}{T+1 / 2}\right)$, we conclude that

$$
0<\frac{\sin \frac{T+1-k}{2 T+1} \pi}{2 \frac{T}{k} \cos \frac{\theta_{\varphi, k}}{2}}<\frac{1}{2 \frac{T}{k} \cos \frac{(T+1-k) \pi}{2 T+1}} \leq \infty .
$$

This, together with (B.32), implies that

$$
\tan T \varpi_{k}=O\left(\frac{T}{k}(1-\varphi)\right)
$$

It follows that

$$
\varpi_{k}=O\left(\frac{1-\varphi}{k}\right) .
$$

(B.23)-(B.25), together with the assumption (A8), (B.35) and (B.6), imply that when $T$ is big enough there exists a constant $\Upsilon>0$ such that

$$
\frac{\lambda_{1, k}-\lambda_{\varphi, k}}{\lambda_{1, k}} \leq \Upsilon T(1-\varphi)
$$

for any $k$.

Lemmas 7 can be verified with some straightforward computations and the simple fact that

$$
\sin (k+j) \theta+\sin (k-j) \theta=2 \sin k \theta \cos j \theta .
$$

We ignore the details here.

Proof of Lemma 8: From (B.8) we obtain

$$
\left|x_{k, j}\right| \leq 1
$$

Lemma 8 can be then proved with some straightforward computations. We ignore details here.

Proof of Lemma 9:

Let's prove (B.13) at first. Note that

$$
\left|\left(a_{0}+2 \sum_{1 \leq j \leq T-1} a_{j}(-1)^{j} \cos \left(j \theta_{\varphi, k}\right)\right)-\left(a_{0}+2 \sum_{1 \leq j \leq \infty} a_{j}\right)\right| \leq 2 \sum_{1 \leq j \leq T-1}\left|a_{j}\right|\left|\cos \left(j\left(\pi-\theta_{\varphi, k}\right)\right)-1\right|+2 \sum_{T \leq j}\left|a_{j}\right| .
$$

For a fixed $k$, we can find a $j_{k}$ to satisfy $\frac{\pi}{3} \leq j\left(\pi-\theta_{\varphi, k}\right) \leq \frac{\pi}{2}$. It follows that

$$
\begin{aligned}
2 \sum_{1 \leq j \leq j_{k}}\left|a_{j}\right|\left|\cos \left(j\left(\pi-\theta_{\varphi, k}\right)\right)-1\right| & \leq \frac{1}{2} \sum_{1 \leq j \leq j_{k}}\left|a_{j}\right| j^{2}\left(\pi-\theta_{\varphi, k}\right)^{2} \\
& \leq \frac{j_{k}\left(\pi-\theta_{\varphi, k}\right)^{2}}{2} \sum_{1 \leq j \leq j_{k}} j\left|a_{j}\right| \\
& \leq \frac{k \pi^{2}}{4(T+1)} \sum_{1 \leq j \leq \infty} j\left|a_{j}\right|
\end{aligned}
$$


and that

$$
\begin{aligned}
& 2 \sum_{j_{k}<j \leq T-1}\left|a_{j}\right|\left|\cos \left(j\left(\pi-\theta_{\varphi, k}\right)\right)-1\right|+2 \sum_{T \leq j}\left|a_{j}\right| \leq 4 \sum_{j \geq j_{k}}\left|a_{j}\right| \\
& \leq j_{k}^{-1} 4 \sum_{j \geq j_{k}} j\left|a_{j}\right| \leq \frac{3(2 k-1)}{2 T+1} \sum_{1 \leq j \leq \infty} j\left|a_{j}\right| .
\end{aligned}
$$

The assumption (A1) implies that

$$
\sum_{i=0}^{\infty} i\left|a_{i}\right|<\infty
$$

From the assumption (A2), (B.41) and truncation conditions we can find

$$
\lim _{T \rightarrow \infty}\left(a_{0}+2 \sum_{1 \leq j \leq T-1} a_{j}(-1)^{j} \cos \left(j \theta_{\varphi, k}\right)\right)=\lim _{T \rightarrow \infty}\left(a_{0}+2 \sum_{1 \leq j \leq \infty} a_{j}\right)=\left(\sum_{i=0}^{\infty} b_{i}\right)^{2}=s^{2}>0 .
$$

In view of (B.5), (B.12) and (B.42), we have (B.13).

Now we consider the eigenvalues of $\mathbf{A C}_{\varphi}^{*} \mathbf{C}_{\varphi}$. From Lemmas 4-8, we can write $\mathbf{C}_{\varphi}^{*} \mathbf{C}_{\varphi}$ as $\mathbf{V}_{\varphi}^{*} \Lambda_{\varphi}^{1 / 2} \Lambda_{\varphi}^{1 / 2} \mathbf{V}_{\varphi}$, where

$$
\boldsymbol{\Lambda}_{\varphi}=\operatorname{diag}\left\{\lambda_{\varphi, 1}, \cdots, \lambda_{\varphi, T}\right\}, \mathbf{V}_{\varphi}^{*}=\left(\tilde{\mathbf{y}}_{\mathbf{1}}, \cdots, \tilde{\mathbf{y}}_{\mathbf{T}}\right) .
$$

Then we just need to consider the eigenvalues of $\mathbf{M}_{\mathbf{a}, \varphi}=\boldsymbol{\Lambda}_{\varphi}^{\mathbf{1} / \mathbf{2}} \mathbf{V}_{\varphi} \mathbf{A} \mathbf{V}_{\varphi}^{*} \boldsymbol{\Lambda}_{\varphi}^{\mathbf{1} / \mathbf{2}}$. Define the (i,j) entry of $\mathbf{M}_{\mathbf{a}, \varphi}$ as $\grave{M}_{i, j}$. Then

and

$$
\grave{M}_{i, j}=\lambda_{\varphi, i}^{1 / 2} \lambda_{\varphi, j}^{1 / 2} \tilde{\mathbf{y}}_{\mathbf{i}}^{*} A \tilde{\mathbf{y}}_{\mathbf{j}}=\frac{\lambda_{\varphi, i}^{1 / 2} \lambda_{\varphi, j}^{1 / 2}}{\left\|\tilde{\mathbf{x}}_{\mathbf{i}}\right\|\left\|\tilde{\mathbf{x}}_{\mathbf{j}}\right\|} \tilde{\mathbf{x}}_{\mathbf{i}}^{*} A \tilde{\mathbf{x}}_{\mathbf{j}}
$$

$$
\tilde{\mathbf{x}}_{\mathbf{i}}^{*} A \tilde{\mathbf{x}}_{\mathbf{j}}=a_{0} \sum_{h=1}^{T} x_{i h} x_{j h}+\sum_{h=1}^{T-1} a_{h}\left(\sum_{f=1}^{T-h} x_{i f} x_{j, f+h}+\sum_{f=h+1}^{T} x_{i f} x_{j, f-h}\right) .
$$

Note that $x_{j, f-h}+x_{j, f+h}=2 *(-1)^{h} x_{j f} \cos \left(h \theta_{\varphi, j}\right)$. Then

$$
\tilde{\mathbf{x}}_{\mathbf{i}}^{*} A \tilde{\mathbf{x}}_{\mathbf{j}}=\left(a_{0}+2 \sum_{h=1}^{T-1} a_{h}(-1)^{h} \cos \left(h \theta_{\varphi, j}\right)\right)\left(\sum_{h=1}^{T} x_{i h} x_{j h}\right)-\sum_{h=1}^{T-1} a_{h}\left(\sum_{f=T-h+1}^{T} x_{i f} x_{j, f+h}+\sum_{f=1}^{h} x_{i f} x_{j, f-h}\right) .
$$

Note that $\sum_{h=1}^{T} x_{i h} x_{j h}=1\{i=j\}\left\|\tilde{\mathbf{x}}_{\mathbf{i}}\right\|\left\|\tilde{\mathbf{x}}_{\mathbf{j}}\right\|$ and

$$
\left|\sum_{h=1}^{T-1} a_{h}\left(\sum_{f=T-h+1}^{T} x_{i f} x_{j, f+h}+\sum_{f=1}^{h} x_{i f} x_{j, f-h}\right)\right| \leq 2 \sum_{h=1}^{T-1} h\left|a_{h}\right|
$$

Then we can write

$$
\begin{aligned}
& \mathbf{M}_{\mathbf{a}, \varphi}=\mathbf{M}_{\mathbf{a}, \varphi, \mathbf{R}}+\operatorname{diag}\left\{\gamma_{\varphi, 1}, \cdots, \gamma_{\varphi, T}\right\}=\mathbf{M}_{\mathbf{a}, \varphi, \mathbf{R}}+\boldsymbol{\Lambda}_{\mathbf{a}, \varphi, \mathbf{M}} . \\
& \left\|\mathbf{M}_{\mathbf{a}, \varphi, \mathbf{R}}\right\|_{2}^{2} \leq \sum_{i=1}^{T} \sum_{j=1}^{T} \frac{\lambda_{\varphi, i} \lambda_{\varphi, j}}{\left\|\tilde{\mathbf{x}}_{\mathbf{i}}\right\|^{2}\left\|\tilde{\mathbf{x}}_{\mathbf{j}}\right\|^{2}} *\left(2 \sum_{h=1}^{T-1} h\left|a_{h}\right|\right)^{2}=O\left(\frac{\lambda_{\varphi, 1}^{2}}{T^{2}}\right) .
\end{aligned}
$$

This, together with (B.13), leads to (B.14).

Proof of Lemma 12: From (B.7) we can find that

$$
\left|\sum_{k>k_{0}} \beta_{\varphi, k}\right|<(1+\Upsilon T(1-\varphi))\left|\sum_{k>k_{0}} \beta_{1, k}\right| .
$$

This, together with (B.6) and (B.16), implies (B.17). 


\section{B.2 Eigenvectors of $\mathrm{C}_{\varphi} \mathrm{AC}_{\varphi}^{*}$}

This section is to investigate the eigenvectors of $\mathbf{C}_{\varphi} \mathbf{A} \mathbf{C}_{\varphi}^{*}$. Recalling (B.10), we normalize $\left\{\tilde{\mathbf{x}}_{\mathbf{k}}\right\}_{1 \leq k \leq T}$ to get $\left\{\tilde{\mathbf{y}}_{\mathbf{k}}\right\}_{1 \leq k \leq T}$. Then we study the eigenvectors of of $\mathbf{A C}_{\varphi}^{*} \mathbf{C}_{\varphi}$ by representing them with $\left\{\tilde{\mathbf{y}}_{\mathbf{k}}\right\}_{1 \leq k \leq T}$. At last we give some result about the eigenvectors of $\mathbf{C}_{\varphi} \mathbf{A C}_{\varphi}^{*}$ which is necessary for the future proof. Our results are the following.

Lemma 13. Let $\left\{\mathbf{u}_{\mathbf{k}}\right\}_{1 \leq k \leq T}$ be orthogonal and real vectors such that $\left\|\mathbf{u}_{k}\right\|=1$ and

$$
\mathbf{C}_{\varphi} \mathbf{A} \mathbf{C}_{\varphi}^{*} \mathbf{u}_{\mathbf{k}}=\beta_{\varphi, k} \mathbf{u}_{\mathbf{k}}
$$

Define $\mathbf{f}_{\mathbf{k}}=\frac{\mathbf{C}_{\varphi}^{-1} \mathbf{u}_{\mathbf{k}}}{\left\|\mathbf{C}_{\varphi}^{-1} \mathbf{u}_{\mathbf{k}}\right\|}$ such that

$$
\mathbf{f}_{\mathbf{k}}=\Sigma_{j=1}^{T} \alpha_{k j} \mathbf{y}_{\mathbf{j}}
$$

with

$$
\Sigma_{j=1}^{T} \alpha_{k j}^{2}=1
$$

Then when $k \geq 1$ is fixed,

$$
\frac{\alpha_{k k}^{2} \lambda_{\varphi, k}}{\Sigma_{j=1}^{T} \alpha_{k j}^{2} \lambda_{\varphi, j}}=1+O\left(T^{-1}\right),
$$

where $\left\{\lambda_{\varphi, j}\right\}$ are given in Lemma 4 .

Lemma 14. Let $\left(S_{k, 1}, \cdots, S_{k, T+l}\right)^{\prime}=\mathbf{s}_{\mathbf{k}}=\frac{\mathbf{F}^{*} \mathbf{C}_{\varphi}^{*} \mathbf{u}_{\mathbf{k}}}{\sqrt{\gamma_{\varphi, 1}}}$. Then $\left\{\mathbf{s}_{\mathbf{k}}\right\}_{1 \leq k \leq T}$ are orthogonal and

$$
\sum_{j=1}^{T+l} S_{k, j}^{4}=O\left(T^{-1}\right)
$$

Proof of Lemma 13: From $\mathbf{f}_{\mathbf{k}}=\frac{\mathbf{C}_{\varphi}^{-1} \mathbf{u}_{\mathbf{k}}}{\left\|\mathbf{C}^{-1} \mathbf{u}_{\mathbf{k}}\right\|}$ and (B.48), we have $\left\|\mathbf{f}_{\mathbf{k}}\right\|=1$ and

$$
\mathbf{A C}_{\varphi}^{*} \mathbf{C}_{\varphi} \mathbf{f}_{\mathbf{k}}=\beta_{\varphi, k} \mathbf{f}_{\mathbf{k}}
$$

From (B.45) and (B.53), we have

$$
\beta_{\varphi, k}=\frac{\mathbf{f}_{\mathbf{k}}^{*} \mathbf{C}_{\varphi}^{*} \mathbf{C}_{\varphi} \mathbf{A} \mathbf{C}_{\varphi}^{*} \mathbf{C}_{\varphi} \mathbf{f}_{\mathbf{k}}}{\left\|\mathbf{C}_{\varphi} \mathbf{f}_{\mathbf{k}}\right\|^{2}}=\frac{\mathbf{f}_{\mathbf{k}}^{*} \mathbf{V}_{\varphi}^{*} \boldsymbol{\Lambda}_{\varphi}^{\mathbf{1} / \mathbf{2}}\left(\boldsymbol{\Lambda}_{\mathbf{a}, \varphi, \mathbf{M}}+\mathbf{M}_{\mathbf{a}, \varphi, \mathbf{R}}\right) \boldsymbol{\Lambda}_{\varphi}^{\mathbf{1} / \mathbf{2}} \mathbf{V}_{\varphi} \mathbf{f}_{\mathbf{k}}}{\left\|\mathbf{C}_{\varphi} \mathbf{f}_{\mathbf{k}}\right\|^{2}}
$$

It follows that

$$
\frac{\left|\mathbf{f}_{\mathbf{k}}^{*} \mathbf{V}_{\varphi}^{*} \Lambda_{\varphi}^{\mathbf{1} / \mathbf{2}} \boldsymbol{\Lambda}_{\mathbf{a}, \varphi, \mathbf{M}} \boldsymbol{\Lambda}_{\varphi}^{\mathbf{1} / 2} \mathbf{V}_{\varphi} \mathbf{f}_{\mathbf{k}}\right|-\left|\mathbf{f}_{\mathbf{k}}^{*} \mathbf{V}_{\varphi}^{*} \Lambda_{\varphi}^{\mathbf{1} / \mathbf{2}} \mathbf{M}_{\mathbf{a}, \varphi, \mathbf{R}} \boldsymbol{\Lambda}_{\varphi}^{\mathbf{1} / \mathbf{2}} \mathbf{V}_{\varphi} \mathbf{f}_{\mathbf{k}}\right|}{\left\|\mathbf{C}_{\varphi} \mathbf{f}_{\mathbf{k}}\right\|^{2}} \leq \beta_{\varphi, k}
$$

and

$$
\beta_{\varphi, k} \leq \frac{\left|\mathbf{f}_{\mathbf{k}}^{*} \mathbf{V}_{\varphi}^{*} \boldsymbol{\Lambda}_{\varphi}^{\mathbf{1} / \mathbf{2}} \boldsymbol{\Lambda}_{\mathbf{a}, \varphi, \mathbf{M}} \boldsymbol{\Lambda}_{\varphi}^{\mathbf{1} / \mathbf{2}} \mathbf{V}_{\varphi} \mathbf{f}_{\mathbf{k}}\right|+\left|\mathbf{f}_{\mathbf{k}}^{*} \mathbf{V}_{\varphi}^{*} \boldsymbol{\Lambda}_{\varphi}^{\mathbf{1} / \mathbf{2}} \mathbf{M}_{\mathbf{a}, \varphi, \mathbf{R}} \boldsymbol{\Lambda}_{\varphi}^{\mathbf{1} / \mathbf{2}} \mathbf{V}_{\varphi} \mathbf{f}_{\mathbf{k}}\right|}{\left\|\mathbf{C}_{\varphi} \mathbf{f}_{\mathbf{k}}\right\|^{2}} .
$$

By (B.9), (B.10), (B.43) and (B.49), we have

$$
\left\|\mathbf{C}_{\varphi} \mathbf{f}_{\mathbf{k}}\right\|=\sqrt{\Sigma_{j=1}^{T} \alpha_{k j}^{2} \lambda_{\varphi, j}} .
$$

(B.9), (B.10), (B.45) and (B.49) imply that

$$
\mathbf{f}_{\mathbf{k}}^{*} \mathbf{V}_{\varphi}^{*} \boldsymbol{\Lambda}_{\varphi}^{\mathbf{1} / \mathbf{2}} \boldsymbol{\Lambda}_{\mathbf{a}, \varphi, \mathbf{M}} \boldsymbol{\Lambda}_{\varphi}^{\mathbf{1} / \mathbf{2}} \mathbf{V}_{\varphi} \mathbf{f}_{\mathbf{k}}=\Sigma_{j=1}^{T} \alpha_{k j}^{2} \gamma_{\varphi, j} \lambda_{\varphi, j}
$$


From (B.46), we have

$$
\frac{\left|\mathbf{f}_{\mathbf{k}}^{*} \mathbf{V}_{\varphi}^{*} \boldsymbol{\Lambda}_{\varphi}^{\mathbf{1} / \mathbf{2}} \mathbf{M}_{\mathbf{a}, \varphi, \mathbf{R}} \boldsymbol{\Lambda}_{\varphi}^{\mathbf{1} / \mathbf{2}} \mathbf{V}_{\varphi} \mathbf{f}_{\mathbf{k}}\right|}{\left\|\mathbf{C}_{\varphi} \mathbf{f}_{\mathbf{k}}\right\|^{2}} \leq\left\|\mathbf{M}_{\mathbf{a}, \varphi, \mathbf{R}}\right\|_{2}=O(T)
$$

This, together with (B.54)-(B.57), implies that

$$
\frac{\Sigma_{j=1}^{T} \alpha_{k j}^{2} \gamma_{\varphi, j} \lambda_{\varphi, j}}{\Sigma_{j=1}^{T} \alpha_{k j}^{2} \lambda_{\varphi, j}}-O(T) \leq \beta_{\varphi, k} \leq \frac{\Sigma_{j=1}^{T} \alpha_{k j}^{2} \gamma_{\varphi, j} \lambda_{\varphi, j}}{\Sigma_{j=1}^{T} \alpha_{k j}^{2} \lambda_{\varphi, j}}+O(T) .
$$

By Lemma 9, for any fixed $k$ we have

$$
\Sigma_{j=1}^{T} \frac{\alpha_{k j}^{2} \lambda_{\varphi, j}}{\sum_{j=1}^{T} \alpha_{k j}^{2} \lambda_{\varphi, j}} \frac{\gamma_{\varphi, j}}{\beta_{\varphi, k}}-O\left(T^{-1}\right) \leq 1 \leq \Sigma_{j=1}^{T} \frac{\alpha_{k j}^{2} \lambda_{\varphi, j}}{\sum_{j=1}^{T} \alpha_{k j}^{2} \lambda_{\varphi, j}} \frac{\gamma_{\varphi, j}}{\beta_{\varphi, k}}+O\left(T^{-1}\right) .
$$

Note that $\left\{\mathbf{u}_{\mathbf{k}}\right\}_{1 \leq k \leq T}$ are orthogonal and $\left\{\tilde{\mathbf{y}}_{\mathbf{k}}\right\}_{1 \leq k \leq T}$ are orthogonal. When $k \neq m$, from (B.9), (B.10) and (B.49) we have

$$
0=\mathbf{u}_{\mathbf{k}}^{*} \mathbf{u}_{\mathbf{m}}=\frac{\mathbf{f}_{\mathbf{k}}^{*} \mathbf{C}_{\varphi}^{*} \mathbf{C}_{\varphi} \mathbf{f}_{\mathbf{m}}}{\left\|\mathbf{C}_{\varphi} \mathbf{f}_{\mathbf{k}}\right\|\left\|\mathbf{C}_{\varphi} \mathbf{f}_{\mathbf{m}}\right\|}=\frac{\Sigma_{j=1}^{T} \alpha_{k j} \alpha_{m j} \lambda_{\varphi, j}}{\left\|\mathbf{C}_{\varphi} \mathbf{f}_{\mathbf{k}}\right\|\left\|\mathbf{C}_{\varphi} \mathbf{f}_{\mathbf{m}}\right\|}
$$

This implies that

$$
\Sigma_{j=1}^{T} \alpha_{k j} \alpha_{m j} \lambda_{\varphi, j}=0
$$

Moreover let $v_{k j}=\frac{\alpha_{k j} \sqrt{\lambda_{\varphi, j}}}{\sqrt{\sum_{j=1}^{T} \alpha_{k j}^{2} \lambda_{\varphi, j}}}$. We have

$$
\Sigma_{j=1}^{T} v_{k j}^{2}=1
$$

Note that (B.58) is equivalent to

$$
\Sigma_{j=1}^{T} v_{k j}^{2} \frac{\gamma_{\varphi, j}}{\beta_{\varphi, k}}-O\left(T^{-1}\right) \leq 1 \leq \Sigma_{j=1}^{T} v_{k j}^{2} \frac{\gamma_{\varphi, j}}{\beta_{\varphi, k}}+O\left(T^{-1}\right)
$$

Also (B.59) implies that

$$
\Sigma_{j=1}^{T} v_{k j} v_{m j}=0 .
$$

We consider $v_{k j}$ for fixed $k$ below. When $k=1$ and $T$ is big enough, Lemma 9, (B.60) and (B.61) imply

$$
O\left(T^{-1}\right)=\left|1-\Sigma_{j=1}^{T} v_{1 j}^{2} \frac{\gamma_{\varphi, j}}{\beta_{\varphi, 1}}\right| \geq\left(1-v_{11}^{2}\right) \frac{\beta_{\varphi, 1}-\gamma_{\varphi, 2}}{\beta_{\varphi, 1}}-v_{11}^{2} \frac{\left|\beta_{\varphi, 1}-\gamma_{\varphi, 1}\right|}{\beta_{\varphi, 1}} .
$$

In view of (B.13)-(B.14), we have $\frac{\beta_{\varphi, 1}-\gamma_{\varphi, 1}}{\beta_{\varphi, 1}}=O\left(T^{-1}\right)$. Recalling that $\theta_{\varphi, k} \in\left[\frac{(T+1-k) \pi}{T+1}, \frac{(T+1-k) \pi}{T+1 / 2}\right]$, we can find that $\left|\frac{\beta_{\varphi, 1}}{\beta_{\varphi, 1}-\gamma_{\varphi, 2}}\right|$ is bounded. It follows that (B.63) implies that $v_{11}^{2}=1+O\left(T^{-1}\right)$ and $\Sigma_{j=2}^{T} v_{1 j}^{2}=O\left(T^{-1}\right)$. From (B.62), for any $k \neq 1$ we have

$$
\left|v_{k 1} v_{11}\right|=\left|\Sigma_{j=2}^{T} v_{k j} v_{1 j}\right| \leq \sqrt{\Sigma_{j=2}^{T} v_{k j}^{2}} \sqrt{\Sigma_{j=2}^{T} v_{1 j}^{2}}=O\left(T^{-1 / 2}\right) .
$$

This implies $v_{k 1}^{2}=O\left(T^{-1}\right)$. It's similar to obtain that $v_{22}^{2}=1+O\left(T^{-1}\right)$ and $v_{k 2}^{2}=O\left(T^{-1}\right)$ for any $k \neq 2$.

By repeating these steps we conclude that $v_{k k}^{2}=1+O\left(T^{-1}\right)$ for any fixed $k$. This implies (B.51). 
Proof of Lemma 14: Note that $\left\{\mathbf{s}_{\mathbf{k}}\right\}_{1 \leq k \leq T}$ are orthogonal and real due to orthogonality of $\left\{\mathbf{u}_{\mathbf{k}}\right\}_{1 \leq k \leq T}$. We conclude from (B.9) and (B.49) that

$$
\mathbf{s}_{\mathbf{k}}=\frac{\mathbf{F}^{*} \mathbf{C}_{\varphi}^{*} \mathbf{C}_{\varphi} \mathbf{f}_{\mathbf{k}}}{\sqrt{\gamma_{\varphi, 1}}\left\|\mathbf{C}_{\varphi} \mathbf{f}_{\mathbf{k}}\right\|}=\frac{1}{\sqrt{\gamma_{\varphi, 1}}\left\|\mathbf{C}_{\varphi} \mathbf{f}_{\mathbf{k}}\right\|} \Sigma_{j=1}^{T} \alpha_{k j} \lambda_{\varphi, j} \mathbf{F}^{*} \tilde{\mathbf{y}}_{\mathbf{j}}=\mathbf{s}_{\mathbf{k}, \mathbf{M}}+\mathbf{s}_{\mathbf{k}, \mathbf{R}}
$$

where

$$
\mathbf{s}_{\mathbf{k}, \mathbf{M}}=\frac{1}{\sqrt{\gamma_{\varphi, 1}}\left\|\mathbf{C}_{\varphi} \mathbf{f}_{\mathbf{k}}\right\|} \alpha_{k k} \lambda_{\varphi, k} \mathbf{F}^{*} \tilde{\mathbf{y}}_{\mathbf{k}}, \quad \mathbf{s}_{\mathbf{k}, \mathbf{R}}=\frac{1}{\sqrt{\gamma_{\varphi, 1}}\left\|C_{\varphi} f_{k}\right\|} \Sigma_{j \neq k} \alpha_{k j} \lambda_{j} \mathbf{F}^{*} \tilde{\mathbf{y}}_{\mathbf{j}}
$$

By Hölder's inequality, we have

$$
\left\|\mathbf{s}_{\mathbf{k}, \mathbf{R}}\right\|=\left\|\frac{1}{\sqrt{\gamma_{\varphi, 1}}\left\|\mathbf{C}_{\varphi} \mathbf{f}_{\mathbf{k}}\right\|} \Sigma_{j \neq k} \alpha_{k j} \lambda_{\varphi, j} \mathbf{F}^{*} \tilde{\mathbf{y}}_{\mathbf{j}}\right\| \leq \frac{1}{\sqrt{\gamma_{\varphi, 1}}\left\|\mathbf{C}_{\varphi} \mathbf{f}_{\mathbf{k}}\right\|}\|\mathbf{F}\|_{2} \sqrt{\Sigma_{j \neq k} \alpha_{k j}^{2} \lambda_{\varphi, j}^{2}}
$$

Recalling $\mathbf{A}=\mathbf{F F}^{*}$, we have

$$
\|\mathbf{F}\|_{2}=\sqrt{\|\mathbf{A}\|_{2}}
$$

Since $A$ is a Hermitian Toeplitz matrix, from [18],

$$
\|\mathbf{A}\|_{2} \leq 2 \sum_{0 \leq k \leq l}\left|a_{k}\right|
$$

By (B.41) we can get

$$
\|\mathbf{F}\|_{2}=\sqrt{\|\mathbf{A}\|_{2}}<\infty
$$

From Lemma 5, (B.51), and (B.56) we can obtain that for any fixed $k$,

$$
\frac{\sqrt{\Sigma_{j \neq k} \alpha_{k j}^{2} \lambda_{\varphi, j}^{2}}}{\left\|\mathbf{C}_{\varphi} \mathbf{f}_{\mathbf{k}}\right\|} \leq \sqrt{\frac{\sum_{j \neq k} \alpha_{k j}^{2} \lambda_{\varphi, j}}{\sum_{j=1}^{T} \alpha_{k j}^{2} \lambda_{\varphi, j}}} \sqrt{\lambda_{\varphi, 1}}=O\left(T^{1 / 2}\right) .
$$

This, together with (B.13), implies that for any fixed $k$,

$$
\left\|\mathbf{s}_{\mathbf{k}, \mathbf{R}}\right\|=O\left(T^{-1 / 2}\right)
$$

Similarly, we can also obtain that $\frac{1}{\sqrt{\gamma_{1}}\left\|\mathbf{C f}_{\mathbf{k}}\right\|} \alpha_{k k} \lambda_{k}$ is bounded for any fixed $k$.

Let $S_{k, M, j}$ be the $j$ th element of $\mathbf{s}_{\mathbf{k}, \mathbf{M}}$ and $S_{k, R, j}$ be the $j$ th element of $\mathbf{s}_{\mathbf{k}, \mathbf{R}}$. From (B.11), (B.51) and (B.66) and the assumption (A1) we can obtain that for any fixed $k$,

$$
\left|S_{k, M, j}\right| \leq \frac{1}{\sqrt{\gamma_{\varphi, 1}}\left\|\mathbf{C}_{\varphi} \mathbf{f}_{\mathbf{k}}\right\|}\left|\alpha_{k k}\right| \lambda_{k} \frac{2}{\sqrt{2 T+1}} \sum_{h=0}^{l}\left|b_{h}\right|=O\left(T^{-1 / 2}\right) .
$$

It follows from (B.65)-(B.68) that for any fixed $k$,

$$
\begin{aligned}
& \sum_{j=1}^{T+l} S_{k, j}^{4} \leq 8 \sum_{j=1}^{T+l}\left(S_{k, R, j}^{4}+S_{k, M, j}^{4}\right) \\
& \leq 8 \sum_{j=1}^{T+l} S_{k, M, j}^{4}+8\left(\sum_{j=1}^{T+l} S_{k, R, j}^{2}\right)^{2}=O\left(T^{-1}\right) .
\end{aligned}
$$




\section{B.3 The proof of Lemma 1 for truncated matrices}

The proof of the remaining part of the Lemma 1 for truncated matrices is the same as that of the part C.2.3-C.2.4 in [31].

\section{The proof of Lemmas 1-3}

\section{C.1 The proof of Lemma 1}

We can deal with $\frac{1}{p} \mathbf{C}_{\varphi} \mathbf{Y} \boldsymbol{\Sigma}^{\mathbf{1} / \mathbf{2}}(\mathbf{I}-\mathbf{H}) \boldsymbol{\Sigma}^{\mathbf{1} / \mathbf{2}} \mathbf{Y}^{*} \mathbf{C}_{\varphi}^{*}+\frac{1}{p} \mathbf{C}_{\varphi} \mathbf{Y} \boldsymbol{\Sigma}^{\mathbf{1} / \mathbf{2}}(\mathbf{I}-\mathbf{H}) \dot{\mathbf{X}}_{\mathbf{0}} \mathbf{C}_{\varphi, \mathbf{0}}{ }^{*}+\frac{1}{p} \mathbf{C}_{\varphi, \mathbf{0}} \dot{\mathbf{X}}_{\mathbf{0}}(\mathbf{I}-\mathbf{H}) \boldsymbol{\Sigma}^{1 / 2} \mathbf{Y}^{*} \mathbf{C}_{\varphi}^{*}+$ $\frac{1}{p} \mathbf{C}_{\varphi, \mathbf{0}} \dot{\mathbf{X}}_{\mathbf{0}}(\mathbf{I}-\mathbf{H}) \dot{\mathbf{X}}_{\mathbf{0}}^{*} \mathbf{C}_{\varphi, \mathbf{0}}^{*}$ by the same methods of Appendix B in [31]. For the part about $\boldsymbol{\Delta}$, assumption A8 ensures that $\frac{\left|\frac{1}{\sqrt{p}} \boldsymbol{\Delta}(\mathbf{I}-\mathbf{H}) \boldsymbol{\Delta}^{*}\right|_{2}}{T^{2}} \leq \frac{\frac{1}{\sqrt{p}} \operatorname{trace}\left(\boldsymbol{\Delta}(\mathbf{I}-\mathbf{H}) \boldsymbol{\Delta}^{*}\right)}{T^{2}}=o_{p}(1)$. The other part is similar.

\section{C.2 The proof of Lemmas 2-3}

At first we consider the impact of $\delta_{\mathbf{t}}$. Recalling the definition of $\grave{\mu}_{m}$, we write

$$
\begin{aligned}
& \grave{\mu}_{m_{1}}=\sum_{i=2}^{T} \frac{\grave{\mathbf{x}}_{i, i}}{p(T-1)}+2 \sum_{j=1}^{m} \sum_{i=2}^{T-j} \frac{\grave{\mathbf{x}}_{i, i+j}}{p(T-j-1)} \\
& =\sum_{i=2}^{T} \frac{\left(\mathbf{x}_{\mathbf{i}}-\delta_{\mathbf{i}}-\mathbf{x}_{\mathbf{i}-\mathbf{1}}+\delta_{\mathbf{i}-\mathbf{1}}\right)(\mathbf{I}-\mathbf{H})^{\prime}\left(\mathbf{x}_{\mathbf{i}}-\delta_{\mathbf{i}}-\mathbf{x}_{\mathbf{i}-\mathbf{1}}+\delta_{\mathbf{i}-\mathbf{1}}\right)}{p(T-1)} \\
& +2 \sum_{j=1}^{m_{1}} \sum_{i=2}^{T-j} \frac{\left(\mathbf{x}_{\mathbf{i}}-\delta_{\mathbf{i}}-\mathbf{x}_{\mathbf{i}-\mathbf{1}}+\delta_{\mathbf{i}-\mathbf{1}}\right)(\mathbf{I}-\mathbf{H})^{\prime}\left(\mathbf{x}_{\mathbf{i}+\mathbf{j}}-\delta_{\mathbf{i}+\mathbf{j}}-\mathbf{x}_{\mathbf{i}+\mathbf{j}-\mathbf{1}}+\delta_{\mathbf{i}+\mathbf{j}-\mathbf{1}}\right)}{p(T-j-1)} \\
& +\sum_{i=2}^{T} \frac{2\left(\mathbf{x}_{\mathbf{i}}-\delta_{\mathbf{i}}-\mathbf{x}_{\mathbf{i}-\mathbf{1}}+\delta_{\mathbf{i}-\mathbf{1}}\right)(\mathbf{I}-\mathbf{H})^{\prime}\left(\delta_{\mathbf{i}}-\delta_{\mathbf{i}-\mathbf{1}}\right)}{p(T-1)} \\
& +2 \sum_{j=1}^{m_{1}} \sum_{i=2}^{T-j} \frac{\left(\delta_{\mathbf{i}}-\delta_{\mathbf{i}-\mathbf{1}}\right)(\mathbf{I}-\mathbf{H})^{\prime}\left(\mathbf{x}_{\mathbf{i}+\mathbf{j}}-\delta_{\mathbf{i}+\mathbf{j}}-\mathbf{x}_{\mathbf{i}+\mathbf{j}-\mathbf{1}}+\delta_{\mathbf{i}+\mathbf{j}-\mathbf{1}}\right)}{p(T-j-1)} \\
& +2 \sum_{j=1}^{m_{1}} \sum_{i=2}^{T-j} \frac{\left(\mathbf{x}_{\mathbf{i}}-\delta_{\mathbf{i}}-\mathbf{x}_{\mathbf{i}-\mathbf{1}}+\delta_{\mathbf{i}-\mathbf{1}}\right)(\mathbf{I}-\mathbf{H})^{\prime}\left(\delta_{\mathbf{i}+\mathbf{j}}-\delta_{\mathbf{i}+\mathbf{j}-\mathbf{1})}\right)}{p(T-j-1)} \\
& +\sum_{i=2}^{T} \frac{\left(\delta_{\mathbf{i}}-\delta_{\mathbf{i}-\mathbf{1}}\right)(\mathbf{I}-\mathbf{H})^{\prime}\left(\delta_{\mathbf{i}}-\delta_{\mathbf{i}-\mathbf{1}}\right)}{p(T-1)} \\
& +2 \sum_{j=1}^{m_{1}} \sum_{i=2}^{T-j} \frac{\left(\delta_{\mathbf{i}}-\delta_{\mathbf{i}-\mathbf{1}}\right)(\mathbf{I}-\mathbf{H})^{\prime}\left(\delta_{\mathbf{i}+\mathbf{j}}-\delta_{\mathbf{i}+\mathbf{j}-\mathbf{1}}\right)}{p(T-j-1)} .
\end{aligned}
$$

Note that

$$
\lim _{p, T \rightarrow \infty} P(m \leq \min \{q, \sqrt{p}\})=1 .
$$


Moreover

$$
\begin{aligned}
& \left|\sum_{i=2}^{T} \frac{\left(\delta_{\mathbf{i}}-\delta_{\mathbf{i}-\mathbf{1}}\right)(\mathbf{I}-\mathbf{H})^{\prime}\left(\delta_{\mathbf{i}}-\delta_{\mathbf{i}-\mathbf{1}}\right)}{p(T-1)}+2 \sum_{j=1}^{m_{1}} \sum_{i=2}^{T-j} \frac{\left(\delta_{\mathbf{i}}-\delta_{\mathbf{i}-\mathbf{1}}\right)(\mathbf{I}-\mathbf{H})^{\prime}\left(\delta_{\mathbf{i}+\mathbf{j}}-\delta_{\mathbf{i}+\mathbf{j}-\mathbf{1}}\right)}{p(T-j-1)}\right| \\
& \leq\left(2 m_{1}+1\right) \sum_{i=2}^{T} \frac{\left(\delta_{\mathbf{i}}-\delta_{\mathbf{i}-\mathbf{1}}\right)(\mathbf{I}-\mathbf{H})^{\prime}\left(\delta_{\mathbf{i}}-\delta_{\mathbf{i}-\mathbf{1}}\right)}{p\left(T-m_{1}-1\right)} \\
& =\frac{1}{\sqrt{p}} O\left(\sum_{i=2}^{T} \frac{\min \{q, \sqrt{p}\}\left(\delta_{\mathbf{i}}-\delta_{\mathbf{i}-\mathbf{1}}\right)(\mathbf{I}-\mathbf{H})^{\prime}\left(\delta_{\mathbf{i}}-\delta_{\mathbf{i}-\mathbf{1}}\right)}{\sqrt{p} T}\right)=o_{p}\left(\frac{1}{\sqrt{p}}\right),
\end{aligned}
$$

which is ensured by Assumption A11. The other parts involving $\left(\delta_{\mathbf{i}}-\delta_{\mathbf{i}-\mathbf{1}}\right)$ are similar. Note that assumptions $\mathrm{A} 8$ and A10-A11 ensure that $\delta_{\mathbf{t}}$ does not change the asymptotic properties of the estimators. In the following part of this subsection we only consider the case when $\delta_{\mathbf{t}}=\mathbf{0}$.

Proof of Lemma 2: At first by (2.2) we write $\left(\mathbf{x}_{\mathbf{t}}-\mathbf{x}_{\mathbf{t}-\mathbf{1}}\right)$ as follows

$$
\mathbf{x}_{\mathbf{t}}-\mathbf{x}_{\mathbf{t}-\mathbf{1}}=\boldsymbol{\Sigma}^{\mathbf{1} / \mathbf{2}}\left(\mathbf{y}_{\mathbf{t}}+(\varphi-1) \sum_{k=0}^{t-2} \varphi^{k} \mathbf{y}_{\mathbf{t}-\mathbf{k}-\mathbf{1}}+(\varphi-1) \varphi^{t-1} \mathbf{x}_{\mathbf{0}}\right) .
$$

Recalling the assumptions (A7) and (A8) one can find that $E\left\|(\varphi-1) \varphi^{t-1} \mathbf{x}_{\mathbf{0}}\right\|^{2} \rightarrow 0$. Below for facilitating statements we ignore the term and write

$$
\mathbf{x}_{\mathbf{t}}-\mathbf{x}_{\mathbf{t}-\mathbf{1}}=\boldsymbol{\Sigma}^{\mathbf{1} / \mathbf{2}}\left(\mathbf{y}_{\mathbf{t}}+(\varphi-1) \sum_{k=0}^{t-2} \varphi^{k} \mathbf{y}_{\mathbf{t}-\mathbf{k}-\mathbf{1}}\right) .
$$

Recalling the notation $\breve{x}_{f, g}$ below (2.11) we can write $E \breve{\mathbf{x}}_{t, t-j}$ when $j \geq 1$ as

$$
\begin{aligned}
& E \grave{\mathbf{x}}_{t, t-j}=E\left(\mathbf{x}_{\mathbf{t}}-\mathbf{x}_{\mathbf{t}-\mathbf{1}}\right)^{\prime}(\mathbf{I}-\mathbf{H})\left(\mathbf{x}_{\mathbf{t}-\mathbf{j}}-\mathbf{x}_{\mathbf{t}-\mathbf{j}-\mathbf{1}}\right) \\
& =E\left[\left(\mathbf{y}_{\mathbf{t}}+(\varphi-1) \sum_{k=0}^{t-2} \varphi^{k} \mathbf{y}_{\mathbf{t}-\mathbf{k}-\mathbf{1}}\right)^{\prime} \boldsymbol{\Sigma}^{\mathbf{1} / \mathbf{2}}(\mathbf{I}-\mathbf{H}) \boldsymbol{\Sigma}^{\mathbf{1} / \mathbf{2}}\left(\mathbf{y}_{\mathbf{t}-\mathbf{j}}+(\varphi-1) \sum_{k=0}^{t-j-2} \varphi^{k} \mathbf{y}_{\mathbf{t}-\mathbf{j}-\mathbf{k}-\mathbf{1}}\right)\right] \\
& \triangleq \operatorname{tr}\left(\boldsymbol{\Sigma}^{\mathbf{1} / \mathbf{2}}(\mathbf{I}-\mathbf{H}) \boldsymbol{\Sigma}^{\mathbf{1 / 2}}\right)\left(a_{j}+A_{1 t j}+A_{2 t j}+A_{3 t j}+A_{4 t j}+A_{5 t j}+A_{6 t j}\right)
\end{aligned}
$$

where

$$
\begin{aligned}
& A_{1 t j}=(\varphi-1) \sum_{k=0}^{j-1} \varphi^{k} a_{j-k-1}, A_{2 t j}=(\varphi-1) \sum_{k=j}^{t-2} \varphi^{k} a_{k+1-j}, A_{3 t j}=(\varphi-1) \sum_{k=0}^{t-j-2} \varphi^{k} a_{k+j+1}, \\
& A_{4 t j}=(\varphi-1)^{2} \sum_{k_{1}=0}^{j-1} \sum_{k_{2}=0}^{t-j-2} \varphi^{k_{1}+k_{2}} a_{k_{2}+j-k_{1}}, A_{5 t j}=(\varphi-1)^{2} \sum_{k_{1}=j}^{t-2} \sum_{k_{2}=k_{1}-j+1}^{t-j-2} \varphi^{k_{1}+k_{2}} a_{k_{2}+j-k_{1}},
\end{aligned}
$$

and

$$
A_{6 t j}=(\varphi-1)^{2} \sum_{k_{1}=j}^{t-2} \sum_{k_{2}=0}^{k_{1}-j} \varphi^{k_{1}+k_{2}} a_{k_{1}-k_{2}-j}
$$

Hence

$$
\begin{aligned}
& \frac{1}{p(T-j-1)} \sum_{t=j+2}^{T} E \dot{\mathbf{x}}_{t, t-j} \\
& =\frac{\operatorname{tr}\left(\boldsymbol{\Sigma}^{\mathbf{1} / \mathbf{2}}(\mathbf{I}-\mathbf{H}) \boldsymbol{\Sigma}^{\mathbf{1} / \mathbf{2}}\right)}{p} \frac{1}{(T-j-1)} \sum_{t=j+2}^{T}\left(a_{j}+A_{1 t j}+A_{2 t j}+A_{3 t j}+A_{4 t j}+A_{5 t j}+A_{6 t j}(\mathbf{C} .6)\right.
\end{aligned}
$$


We next analyze these term by term.

The terms $A_{k t j}, k=1, \cdots, 6$ can be rewritten as follows:

$$
\begin{aligned}
& \sum_{t=j+2}^{T} A_{2 t j}=\sum_{t=j+2}^{T}(\varphi-1) \sum_{k=1}^{t-1-j} \varphi^{k+j-1} a_{k} \\
& =(\varphi-1) \sum_{k=1}^{T-1-j} \varphi^{k+j-1} \sum_{t=j+1+k}^{T} a_{k}=(\varphi-1) \sum_{k=1}^{T-1-j} \varphi^{k+j-1}(T-j-k) a_{k} ; \\
& \sum_{t=j+2}^{T} A_{3 t j}=\sum_{t=j+2}^{T}(\varphi-1) \sum_{k=j+1}^{t-1} \varphi^{k-j-1} a_{k}=(\varphi-1) \sum_{k=j+1}^{T-1} \varphi^{k-j-1}(T-k) a_{k} \\
& \sum_{t=j+2}^{T} A_{4 t j}=\frac{1-\varphi}{1+\varphi} \sum_{k_{2}=1}^{T-2} a_{k_{2}} \varphi^{\left|j-k_{2}\right|}\left(T-1-\max \left\{k_{2}, j\right\}\right)-\frac{1}{(1+\varphi)^{2}} \sum_{k_{2}=1}^{T-2} a_{k_{2}}\left(\varphi^{2+\left|k_{2}-j\right|}-\varphi^{\min \left\{2+k_{2}+j, 2 T-k_{2}-j\right\}}\right) \\
& -\frac{1-\varphi}{1+\varphi} \sum_{k_{2}=1}^{T-2} a_{k_{2}} \varphi^{k_{2}+j} \max \left\{T-k_{2}-j-1,0\right\}
\end{aligned}
$$



$$
\begin{aligned}
& \text { and } \\
& \sum_{t=j+2}^{T} A_{6 t j}=\frac{1-\varphi}{1+\varphi} \sum_{k_{2}=0}^{T-j-2} a_{k_{2}} \varphi^{j+k_{2}}\left(T-1-j-k_{2}\right)-\frac{1}{(1+\varphi)^{2}} \sum_{k_{2}=0}^{T-j-2} a_{k_{2}}\left(\varphi^{2+k_{2}+j}-\varphi^{2 T-k_{2}-j \gamma \mathrm{C}}\right. \\
& \frac{1}{(T-j-1)} \sum_{t=j+2}^{T} \frac{E \grave{\mathbf{x}}_{t, t-j}}{\operatorname{tr}\left(\boldsymbol{\Sigma}^{\mathbf{1} / \mathbf{2}}(\mathbf{I}-\mathbf{H}) \mathbf{\Sigma}^{\mathbf{1 / 2}}\right)} \\
& =\sum_{k=0}^{T-j-2} a_{k}\left(1\{j=k\}\left(1+\frac{1-\varphi}{1+\varphi}-\frac{1}{(1+\varphi)^{2}} \frac{\varphi^{2}-\varphi^{2 T-2 k}}{T-j-1}\right)\right. \\
& +1\{k<j\}\left((\varphi-1) \varphi^{j-1-k}+\frac{1-\varphi}{1+\varphi} \varphi^{j-k}-\frac{1}{(1+\varphi)^{2}} \frac{\varphi^{2+j-k}-\varphi^{2 T-k-j}}{T-j-1}\right) \\
& +1\{k>j\}\left((\varphi-1) \varphi^{k-1-j} \frac{T-k}{T-1-j}+\frac{1-\varphi}{1+\varphi} \varphi^{k-j} \frac{T-k-1}{T-1-j}-\frac{1}{(1+\varphi)^{2}} \frac{\varphi^{2+k-j}-\varphi^{2 T-k-j}}{T-j-1}\right. \\
& \left.+(\varphi-1) \varphi^{k-1+j} \frac{T-k-j}{T-1-j}+\frac{1-\varphi}{1+\varphi} \varphi^{k+j} \frac{T-k-1-j}{T-1-j}-\frac{1}{(1+\varphi)^{2}} \frac{\varphi^{2+k+j}-\varphi^{2 T-k-j}}{T-j-1}\right) \\
& +O\left((\varphi-1) \sum_{k=T-j-1}^{T-1}\left|a_{k}\right|\right) \text {. }
\end{aligned}
$$

Similarly $E \grave{\mathbf{x}}_{t, t}$ can be written as

$$
\begin{aligned}
& E \grave{\mathbf{x}}_{t, t}=E\left(\mathbf{x}_{\mathbf{t}}-\mathbf{x}_{\mathbf{t}-\mathbf{1}}\right)^{\prime}(\mathbf{I}-\mathbf{H})\left(\mathbf{x}_{\mathbf{t}}-\mathbf{x}_{\mathbf{t}-\mathbf{1}}\right) \\
& =E\left(\mathbf{y}_{\mathbf{t}}+(\varphi-1) \sum_{k=0}^{t-2} \varphi^{k} \mathbf{y}_{\mathbf{t}-\mathbf{k}-\mathbf{1}}\right)^{\prime} \boldsymbol{\Sigma}^{\mathbf{1} / \mathbf{2}}(\mathbf{I}-\mathbf{H}) \boldsymbol{\Sigma}^{\mathbf{1} / \mathbf{2}}\left(\mathbf{y}_{\mathbf{t}-\mathbf{j}}+(\varphi-1) \sum_{k=0}^{t-2} \varphi^{k} \mathbf{y}_{\mathbf{t}-\mathbf{k}-\mathbf{1}}\right) \\
& =\operatorname{tr}\left(\boldsymbol{\Sigma}^{\mathbf{1} / \mathbf{2}}(\mathbf{I}-\mathbf{H}) \boldsymbol{\Sigma}^{\mathbf{1 / 2}}\right)\left(a_{0}+2(\varphi-1) \sum_{k=0}^{t-2} \varphi^{k} a_{k+1}+2 \frac{1-\varphi}{1+\varphi} \sum_{k=1}^{t-2} a_{k}\left(\varphi^{k}-\varphi^{2 t-k-2}\right)\right. \\
& \left.+\frac{1-\varphi}{1+\varphi} a_{0}\left(1-\varphi^{2 t-2}\right)\right) .
\end{aligned}
$$


It follows that

$$
\begin{aligned}
& \frac{1}{p(T-1)} \sum_{t=2}^{T} E \grave{\mathbf{x}}_{t, t} \\
& =\frac{\operatorname{tr}\left(\boldsymbol{\Sigma}^{\mathbf{1} / \mathbf{2}}(\mathbf{I}-\mathbf{H}) \boldsymbol{\Sigma}^{\mathbf{1 / 2}}\right)}{p}\left(a_{0}+\sum_{k=1}^{T-1} \frac{2(\varphi-1) \varphi^{k-1}(T-k)}{T-1} a_{k}+2 \frac{1-\varphi}{1+\varphi} \sum_{k=1}^{T-2} \varphi^{k} a_{k} \frac{T-k-1}{T-1}\right. \\
& \left.-2 \frac{1}{(1+\varphi)^{2}} \sum_{k=1}^{T-2} a_{k} \frac{\varphi^{2+k}-\varphi^{2 T-k}}{T-1}+\frac{1-\varphi}{1+\varphi} a_{0}-\frac{\varphi^{2}-\varphi^{2 T}}{(1+\varphi)^{2}(T-1)} a_{0}\right) .
\end{aligned}
$$

Write $E \grave{\mu}_{m}$ as

$$
E \grave{\mu}_{m}=\frac{\operatorname{tr}\left(\boldsymbol{\Sigma}^{\mathbf{1} / \mathbf{2}}(\mathbf{I}-\mathbf{H}) \boldsymbol{\Sigma}^{\mathbf{1 / 2}}\right)}{p} \sum_{j=0}^{\infty} \kappa_{j} a_{j} .
$$

From (C.12) and (C.14) we conclude that

$$
\begin{aligned}
\kappa_{0} & =1+\frac{1-\varphi}{1+\varphi}-\frac{\varphi^{2}-\varphi^{2 T}}{(1+\varphi)^{2}(T-1)}+2 \sum_{j=1}^{m}\left((\varphi-1) \varphi^{j-1}+\frac{1-\varphi}{1+\varphi} \varphi^{j}-\frac{1}{(1+\varphi)^{2}} \frac{\varphi^{2+j}-\varphi^{2 T-j}}{T-j-1}\right) \\
& =\frac{2 \varphi^{m}}{1+\varphi}-\frac{\varphi^{2}-\varphi^{2 T}}{(1+\varphi)^{2}(T-1)}-2 \sum_{j=1}^{m} \frac{1}{(1+\varphi)^{2}} \frac{\varphi^{2+j}-\varphi^{2 T-j}}{T-1}+2 \sum_{j=1}^{m} \frac{1}{(1+\varphi)^{2}} \frac{j\left(\varphi^{2+j}-\varphi^{2 T-j}\right)}{(T-1)(T-j-1)} \\
& =\frac{2 \varphi^{m}}{1+\varphi}-\frac{\varphi^{2}-\varphi^{2 T}}{(1+\varphi)^{2}(T-1)}-2 \sum_{j=1}^{m} \frac{1}{(1+\varphi)^{2}} \frac{\varphi^{2+j}-\varphi^{2 T-j}}{T-1}+O\left(\frac{m^{2}(1-\varphi)}{T}\right),
\end{aligned}
$$

and

$$
1+2 \sum_{j=1}^{m}(\varphi-1) \varphi^{j-1}<\kappa_{0} \leq 1+\frac{2 \varphi}{1+\varphi} \sum_{j=1}^{m}(\varphi-1) \varphi^{j-1}+\frac{1-\varphi}{1+\varphi}
$$

It follows that

$$
\left|1-\kappa_{0}\right|=O(m(1-\varphi)) .
$$


Similarly, for $1 \leq f \leq m$,

$$
\begin{aligned}
\kappa_{f} / 2= & (\varphi-1) \varphi^{f-1} \frac{T-f}{T-1}+\frac{1-\varphi}{1+\varphi} \varphi^{f} \frac{T-f-1}{T-1}-\frac{\varphi^{2+f}-\varphi^{2 T-f}}{(1+\varphi)^{2}(T-1)} \\
+ & +\frac{1-\varphi}{1+\varphi}-\frac{\varphi^{2}-\varphi^{2 T-2 f}}{(1+\varphi)^{2}(T-f-1)} \\
+ & \sum_{j=f+1}^{m}\left((\varphi-1) \varphi^{j-1-f}+\frac{1-\varphi}{1+\varphi} \varphi^{j-f}-\frac{1}{(1+\varphi)^{2}} \frac{\varphi^{2+j-f}-\varphi^{2 T-j-f}}{T-j-1}\right) \\
+ & \sum_{j=1}^{f-1}\left((\varphi-1) \varphi^{f-1-j} \frac{T-f}{T-1-j}+\frac{1-\varphi}{1+\varphi} \varphi^{f-j} \frac{T-f-1}{T-1-j}-\frac{1}{(1+\varphi)^{2}} \frac{\varphi^{2+f-j}-\varphi^{2 T-j-f}}{T-j-1}\right) \\
+ & \sum_{j=1}^{m}\left((\varphi-1) \varphi^{f-1+j} \frac{T-f-j}{T-1-j}+\frac{1-\varphi}{1+\varphi} \varphi^{f+j} \frac{T-f-j-1}{T-1-j}-\frac{1}{(1+\varphi)^{2}} \frac{\varphi^{2+f+j}-\varphi^{2 T-j-f}}{T-j-1}\right) \\
& -\sum_{j=1}^{m} \frac{1}{(1+\varphi)^{2}} \frac{\varphi^{2+|f-j|}-\varphi^{2 T-j-f}}{T-1}+O\left(\frac{m^{2}(1-\varphi)}{T}\right) \\
= & \kappa_{0}+\frac{\varphi^{m-f}+\varphi^{m+f}-2 \varphi^{m}}{1+\varphi}-\frac{\varphi^{2+f}-\varphi^{2}-\varphi^{2 T-f}+\varphi^{2 T}}{(1+\varphi)^{2}(T-1)} \\
& -\sum_{j=1}^{m} \frac{1}{(1+\varphi)^{2}} \frac{\varphi^{2+j+f}-\varphi^{2+j}-\varphi^{2 T-j-f}+\varphi^{2 T-j}}{T-1} \\
& -\sum_{j=1}^{m} \frac{1}{(1+\varphi)^{2}} \frac{\varphi^{2+|f-j|}-\varphi^{2+j}-\varphi^{2 T-j-f}+\varphi^{2 T-j}}{T-1}+O\left(\frac{m^{2}(1-\varphi)}{T}\right) \\
= & \kappa_{0}+O\left(\frac{m f(1-\varphi)}{T}\right)+O\left(\frac{m^{2}(1-\varphi)}{T}\right) \\
= & \kappa_{0}+o(m(1-\varphi)) .
\end{aligned}
$$

As for $f \geq m_{1}$, we also have

$$
\left|\kappa_{j} / 2\right|=O(m(1-\varphi))
$$

and

$$
\sum_{j=m+1}^{\infty}\left|\kappa_{j}\right|\left|a_{j}\right| \leq \frac{\max _{j>m}\left\{\left|\kappa_{j}\right|\right\}}{m+1} \sum_{j=m+1}^{\infty} j\left|a_{j}\right|=O(1-\varphi)
$$

Then

$$
E \grave{\mu}_{m}=\frac{\operatorname{tr}\left(\boldsymbol{\Sigma}^{\mathbf{1} / \mathbf{2}}(\mathbf{I}-\mathbf{H}) \boldsymbol{\Sigma}^{\mathbf{1 / 2}}\right)}{p} \kappa_{0}\left(a_{0}+2 \sum_{j=1}^{\infty} a_{j}\right)+o(m(1-\varphi))+O(1-\varphi) .
$$

Similarly, one can verify that

$$
\operatorname{Var}\left(\mu_{m}\right)=O\left(\sum_{i=2}^{T} \frac{\mathbf{y}_{\mathbf{i}}^{\prime} \boldsymbol{\Sigma}^{\mathbf{1} / \mathbf{2}}(\mathbf{I}-\mathbf{H}) \boldsymbol{\Sigma}^{\mathbf{1} / \mathbf{2}} \mathbf{y}_{\mathbf{i}}}{p(T-1)}+2 \sum_{j=1}^{m} \sum_{i=2}^{T-j} \frac{\mathbf{y}_{\mathbf{i}}^{\prime} \boldsymbol{\Sigma}^{\mathbf{1} / \mathbf{2}}(\mathbf{I}-\mathbf{H}) \boldsymbol{\Sigma}^{\mathbf{1} / \mathbf{2}} \mathbf{y}_{\mathbf{i}+\mathbf{j}}}{p(T-j-1)}\right) .
$$

This, together with (2.66) in [31], implies

$$
\operatorname{Var}\left(\mu_{m}\right)=o\left(p^{-1}\right) .
$$

Write

$$
\begin{aligned}
& \kappa\left(\theta_{\varphi, 1}\right)-\kappa_{0} \\
& =\varphi^{m}-m \frac{\varphi^{3}-\varphi^{2 T-m}}{2(T-1)}-\frac{2 \varphi^{m}}{1+\varphi}+\frac{\varphi^{2}-\varphi^{2 T}}{(1+\varphi)^{2}(T-1)}+2 \sum_{j=1}^{m} \frac{1}{(1+\varphi)^{2}} \frac{\varphi^{2+j}-\varphi^{2 T-j}}{T-1}+O\left(\frac{m^{2}(1-\varphi)}{T}\right) .
\end{aligned}
$$


Note that

$$
\begin{gathered}
\varphi^{m}-\frac{2 \varphi^{m}}{1+\varphi}=\varphi^{m} \frac{1-\varphi}{1+\varphi}=O(1-\varphi), \\
\frac{\varphi^{2}-\varphi^{2 T}}{(1+\varphi)^{2}(T-1)}=O(1-\varphi), \\
m \frac{\varphi^{3}-\varphi^{2 T-m}}{2(T-1)}-2 \sum_{j=1}^{m} \frac{1}{(1+\varphi)^{2}} \frac{\varphi^{2+j}-\varphi^{2 T-j}}{T-1}=O\left(\frac{m^{2}(1-\varphi)}{T}\right)
\end{gathered}
$$

and

$$
\frac{m^{2}(1-\varphi)}{T}=o(m(1-\varphi))
$$

(C.25)-(C.29) imply (A.4). (C.22)-(C.24) and (A.4) imply (A.5).

Proof of Lemma 3: Now we define

$$
\breve{\mathbf{x}}_{t, s}=\left(\mathbf{x}_{\mathbf{t}}-\mathbf{x}_{\mathbf{t}-1}\right)^{\prime}\left(\mathbf{x}_{\mathbf{s}}-\mathbf{x}_{\mathbf{s}-1}\right) .
$$

We also replace $\grave{\mathbf{x}}_{t, s}$ by $\breve{\mathbf{x}}_{t, s}$ in $\grave{S}_{\sigma^{2}, 0}$ and $\grave{S}_{\sigma^{2}, m}$ and get $S_{\sigma^{2}, 0}$ and $S_{\sigma^{2}, m}$. In fact the differences between $\grave{S}_{\sigma^{2}, 0}$ and $S_{\sigma^{2}, 0}$ are the ones between $\boldsymbol{\Sigma}^{\mathbf{1 / 2}}(\mathbf{I}-\mathbf{H}) \boldsymbol{\Sigma}^{\mathbf{1 / 2}}$ and $\boldsymbol{\Sigma}$. So we just need to study $S_{\sigma^{2}, 0}$ and $S_{\sigma^{2}, m}$ then replace $\boldsymbol{\Sigma}$ by $\boldsymbol{\Sigma}^{\mathbf{1 / 2}}(\mathbf{I}-\mathbf{H}) \boldsymbol{\Sigma}^{\mathbf{1 / 2}}$.

. We rewrite $\left(\mathbf{x}_{\mathbf{t}}-\mathbf{x}_{\mathbf{t}-\mathbf{1}}\right)$ as follows

$$
\begin{aligned}
& \mathbf{x}_{\mathbf{t}}-\mathbf{x}_{\mathbf{t}-\mathbf{1}}=\boldsymbol{\Sigma}^{\mathbf{1} / \mathbf{2}}\left(\mathbf{y}_{\mathbf{t}}+(\varphi-1) \sum_{k=0}^{t-2} \varphi^{k} \mathbf{y}_{\mathbf{t}-\mathbf{k}-\mathbf{1}}\right) \\
& =\boldsymbol{\Sigma}^{\mathbf{1} / \mathbf{2}}\left(\sum_{s=0}^{\infty} b_{s} Z_{t-s}+(\varphi-1) \sum_{k=0}^{t-2} \varphi^{k} \sum_{s=0}^{\infty} b_{s} Z_{t-1-k-s}\right) \\
& =\boldsymbol{\Sigma}^{\mathbf{1} / \mathbf{2}}\left(\sum_{s=-\infty}^{t} b_{t-s} Z_{s}+(\varphi-1) \sum_{k=0}^{t-2} \varphi^{k} \sum_{s=-\infty}^{t-1-k} b_{t-1-k-s} Z_{s}\right) \\
& =\boldsymbol{\Sigma}^{\mathbf{1} / \mathbf{2}}\left(b_{0} Z_{t}+\sum_{s=1}^{t-1}\left(b_{t-s}+(\varphi-1) \sum_{k=0}^{t-1-s} \varphi^{k} b_{t-1-k-s}\right) Z_{s}+\sum_{s=-\infty}^{0}\left(b_{t-s}+(\varphi-1) \sum_{k=0}^{t-2} \varphi^{k} b_{t-1-k-s}\right) Z_{s}\right) .
\end{aligned}
$$

Define a new variable $\breve{b}_{t, s}$ as

$$
\breve{b}_{t, s}= \begin{cases}0 & s>t, \\ b_{0} & s=t, \\ b_{t-s}+(\varphi-1) \sum_{k=0}^{t-1-s} \varphi^{k} b_{t-1-k-s} & 1 \leq s \leq t-1, \\ b_{t-s}+(\varphi-1) \sum_{k=0}^{t-2} \varphi^{k} b_{t-1-k-s} & s \leq 0,\end{cases}
$$

so that

$$
\mathbf{x}_{\mathbf{t}}-\mathbf{x}_{\mathbf{t}-\mathbf{1}}=\boldsymbol{\Sigma}^{\mathbf{1 / 2}} \sum_{s=-\infty}^{t} \breve{b}_{t, s} Z_{s}=\boldsymbol{\Sigma}^{\mathbf{1 / 2}} \sum_{s=-\infty}^{\infty} \breve{b}_{t, s} Z_{s}
$$


Then

$$
\begin{aligned}
& \breve{\mathbf{x}}_{f, g}^{2}=\left(\sum_{i=1}^{p} \sum_{j=1}^{p} \boldsymbol{\Sigma}_{\mathbf{i} \mathbf{j}} \sum_{h_{1}=-\infty}^{\infty} \sum_{h_{2}=-\infty}^{\infty} \breve{b}_{f, h_{1}} \breve{b}_{g, h_{2}} Z_{h_{1}, i} Z_{h_{2}, j}\right)^{2} \\
& =\sum_{i_{1}=1}^{p} \sum_{j_{1}=1}^{p} \sum_{i_{2}=1}^{p} \sum_{j_{2}=1}^{p} \boldsymbol{\Sigma}_{\mathbf{i}_{1} \mathbf{j}_{1}} \boldsymbol{\Sigma}_{\mathbf{i}_{\mathbf{2}} \mathbf{j}_{2}} \sum_{h_{1}=-\infty}^{\infty} \sum_{h_{2}=-\infty}^{\infty} \sum_{h_{3}=-\infty}^{\infty} \sum_{h_{4}=-\infty}^{\infty} \breve{b}_{f, h_{1}} \breve{b}_{g, h_{2}} \breve{b}_{f, h_{3}} \breve{b}_{g, h_{4}} Z_{h_{1}, i_{1}} Z_{h_{2}, j_{1}} Z_{h_{3}, i_{2}} Z_{h_{4}, j_{2}} .
\end{aligned}
$$

Write $S_{\sigma^{2}, 0}=S_{\sigma^{2}, 0, h}+S_{\sigma^{2}, 0, l}$ where

$$
\begin{aligned}
& S_{\sigma^{2}, 0, h}=\frac{1}{\left(T-\frac{3}{2}[T / 2]\right)([T / 2]-1)} \sum_{f=2}^{[T / 2]} \sum_{g=f+[T / 2]}^{T}\left(2 \sum_{i=1}^{p} \sum_{j=1}^{p} \boldsymbol{\Sigma}_{\mathbf{i i}} \Sigma_{\mathbf{i j}} \sum_{h_{1}=-\infty}^{\infty} \sum_{h_{2}=-\infty}^{\infty}\right. \\
& \left.Z_{h_{1}, i}^{3} Z_{h_{2}, j}\left(\breve{b}_{f, h_{1}}^{2} \breve{b}_{g, h_{1}} \breve{b}_{g, h_{2}}+\breve{b}_{f, h_{1}} \breve{b}_{f, h_{2}} \breve{b}_{g, h_{1}}^{2}\right)-3 \sum_{i=1}^{p} \boldsymbol{\Sigma}_{\mathbf{i i}}{ }^{2} \sum_{h=-\infty}^{\infty} Z_{h, i}^{4} \breve{b}_{f, h}^{2} \breve{b}_{g, h}^{2}\right) .
\end{aligned}
$$

Note that when $t>2$

$$
\begin{aligned}
& \sum_{f=2}^{[T / 2]} \sum_{g=f+[T / 2]}^{T} \sum_{i=1}^{p} \sum_{j=1}^{p}\left|\boldsymbol{\Sigma}_{\mathbf{i i}} \boldsymbol{\Sigma}_{\mathbf{i j}}\right| \sum_{h_{1}=-\infty}^{\infty} \sum_{h_{2}=-\infty}^{\infty} \breve{b}_{f, h_{1}}^{2}\left|\breve{b}_{g, h_{1}}\right|\left|\breve{b}_{g, h_{2}}\right| \\
& =\sum_{i=1}^{p} \sum_{j=1}^{p}\left|\boldsymbol{\Sigma}_{\mathbf{i i}} \boldsymbol{\Sigma}_{\mathbf{i j}}\right| \sum_{f=2}^{[T / 2]} \sum_{h_{1}=-\infty}^{f} \breve{b}_{f, h_{1}}^{2} \sum_{g=f+[T / 2]}^{T}\left|\breve{b}_{g, h_{1}}\right| \sum_{h_{2}=-\infty}^{g}\left|\breve{b}_{g, h_{2}}\right| .
\end{aligned}
$$

When $h_{2} \leq g$

$$
\sum_{h_{2}=-\infty}^{g}\left|\breve{b}_{g, h_{2}}\right| \leq 2 \sum_{s=0}^{\infty}\left|b_{s}\right|<\infty
$$

When $h_{1} \leq f \leq g-[T / 2]$

$$
\sum_{g=f+[T / 2]}^{T}\left|\breve{b}_{g, h_{1}}\right| \leq \sum_{s=[T / 2]}^{\infty}\left|b_{s}\right|+\sum_{s=0}^{\infty}\left|b_{s}\right| T(1-\varphi)=o\left(T^{-1}\right)+O(T(1-\varphi))<\infty
$$

and

$$
\sum_{h_{1}=-\infty}^{f} \breve{b}_{f, h_{1}}^{2} \leq\left(\sum_{h_{1}=-\infty}^{f}\left|\breve{b}_{f, h_{1}}\right|\right)^{2}<\infty
$$

Moreover,

$$
\sum_{i=1}^{p} \sum_{j=1}^{p}\left|\boldsymbol{\Sigma}_{\mathbf{i i}} \boldsymbol{\Sigma}_{\mathbf{i j}}\right| \leq \max _{1 \leq i \leq p}\left\{\left|\boldsymbol{\Sigma}_{\mathbf{i i}}\right|\right\} \sqrt{\sum_{i=1}^{p} \sum_{j=1}^{p} \boldsymbol{\Sigma}_{\mathbf{i j}}^{2} p^{2}}=\max _{1 \leq i \leq p}\left\{\left|\boldsymbol{\Sigma}_{\mathbf{i i}}\right|\right\} p \sqrt{\operatorname{tr}\left(\boldsymbol{\Sigma}^{\mathbf{2}}\right)}=O\left(p^{3 / 2}\right) .
$$

From (C.36)-(C.39) we conclude that

$$
\sum_{f=2}^{[T / 2]} \sum_{g=f+[T / 2]}^{T} \sum_{i=1}^{p} \sum_{j=1}^{p}\left|\Sigma_{\mathbf{i i}} \Sigma_{\mathbf{i j}}\right| \sum_{h_{1}=-\infty}^{\infty} \sum_{h_{2}=-\infty}^{\infty} \breve{b}_{f, h_{1}}^{2}\left|\breve{b}_{g, h_{1}}\right|\left|\breve{b}_{g, h_{2}}\right|=O\left(p^{3 / 2} T\right) .
$$

Similarly, we can obtain the order

$$
\sum_{f=2}^{[T / 2]} \sum_{g=f+[T / 2]}^{T} \sum_{i=1}^{p} \sum_{j=1}^{p}\left|\boldsymbol{\Sigma}_{\mathbf{i i}} \Sigma_{\mathbf{i j}}\right| \sum_{h_{1}=-\infty}^{\infty} \sum_{h_{2}=-\infty}^{\infty} \breve{b}_{g, h_{1}}^{2}\left|\breve{b}_{f, h_{1}}\right|\left|\breve{b}_{f, h_{2}}\right|=O\left(p^{3 / 2} T\right)
$$


and

$$
\sum_{i=1}^{p} \boldsymbol{\Sigma}_{\mathbf{i i}}^{2} \sum_{h=-\infty}^{\infty} \breve{b}_{f, h}^{2} \breve{b}_{g, h}^{2}=O(p) .
$$

Summarizing the above we have

$$
E\left|S_{\sigma^{2}, 0, h}\right|=O\left(p^{3 / 2} T^{-1}\right)=o(p) .
$$

Similarly write

$$
\begin{aligned}
E S_{\sigma^{2}, 0, l} & =\frac{1}{\left(T-\frac{3}{2}[T / 2]\right)([T / 2]-1)} \sum_{f=2}^{[T / 2]} \sum_{g=f+[T / 2]}^{T}( \\
& \sum_{i=1}^{p} \sum_{j=1}^{p} \Sigma_{\mathbf{i j}}^{2} \sum_{h_{1}=-\infty}^{\infty} \sum_{h_{2}=-\infty}^{\infty} \breve{b}_{f, h_{1}}^{2} \breve{b}_{g, h_{2}}^{2}+\sum_{i=1}^{p} \sum_{j=1}^{p} \boldsymbol{\Sigma}_{\mathbf{i j}}^{2} \sum_{h_{1}=-\infty}^{\infty} \sum_{h_{2}=-\infty}^{\infty} \breve{b}_{f, h_{1}} \breve{b}_{g, h_{2}} \breve{b}_{f, h_{2}} \breve{b}_{g, h_{1}} \\
& \left.+\sum_{i=1}^{p} \sum_{j=1}^{p} \boldsymbol{\Sigma}_{\mathbf{i i}} \boldsymbol{\Sigma}_{\mathbf{j} \mathbf{j}} \sum_{h_{1}=-\infty}^{\infty} \sum_{h_{2}=-\infty}^{\infty} \breve{b}_{f, h_{1}} \breve{b}_{g, h_{1}} \breve{b}_{f, h_{2}} \breve{b}_{g, h_{2}}-3 \sum_{i=1}^{p} \boldsymbol{\Sigma}_{\mathbf{i i}}^{2} \sum_{h=-\infty}^{\infty} \breve{b}_{f, h}^{2} \breve{b}_{g, h}^{2}\right) .
\end{aligned}
$$

Note that

$$
\left|\sum_{h=-\infty}^{\infty} \breve{b}_{f, h} \breve{b}_{g, h}-a_{g-f}\right|=\left|\sum_{h=-\infty}^{\infty} \breve{b}_{f, h} \breve{b}_{g, h}-\sum_{s=0}^{\infty} b_{s} b_{g-f+s}\right|=O(1-\varphi)
$$

and

$$
\left|\sum_{h=-\infty}^{\infty} \breve{b}_{f, h}^{2}-a_{0}\right|=\left|\sum_{h=-\infty}^{\infty} \breve{b}_{f, h}^{2}-\sum_{s=0}^{\infty} b_{s}^{2}\right|=O(1-\varphi) .
$$

We then conclude that

$$
\frac{\sum_{f=2}^{[T / 2]} \sum_{g=f+[T / 2]}^{T} \sum_{i=1}^{p} \sum_{j=1}^{p} \boldsymbol{\Sigma}_{\mathbf{i j}}^{2} \sum_{h_{1}=-\infty}^{\infty} \sum_{h_{2}=-\infty}^{\infty} \breve{b}_{f, h_{1}}^{2} \breve{b}_{g, h_{2}}^{2}}{\left(T-\frac{3}{2}[T / 2]\right)([T / 2]-1)}=a_{0}^{2} \operatorname{tr}\left(\boldsymbol{\Sigma}^{\mathbf{2}}\right)+O((1-\varphi) p),
$$

$\frac{\sum_{f=2}^{[T / 2]} \sum_{g=f+[T / 2]}^{T} \sum_{i=1}^{p} \sum_{j=1}^{p} \boldsymbol{\Sigma}_{\mathbf{i j}}^{2} \sum_{h_{1}=-\infty}^{\infty} \sum_{h_{2}=-\infty}^{\infty} \breve{b}_{f, h_{1}} \breve{b}_{g, h_{2}} \breve{b}_{f, h_{2}} \breve{b}_{g, h_{1}}}{\left(T-\frac{3}{2}[T / 2]\right)([T / 2]-1)}$

$$
\begin{aligned}
& =\frac{\operatorname{tr}\left(\boldsymbol{\Sigma}^{2}\right)}{\left(T-\frac{3}{2}[T / 2]\right)([T / 2]-1)} \sum_{f=2}^{[T / 2]} \sum_{g=f+[T / 2]}^{T}\left(a_{g-f}^{2}+O\left(a_{g-f}(1-\varphi)\right)+O\left((1-\varphi)^{2}\right)\right) \\
& =O\left(p T^{-3}+p(1-\varphi)^{2}\right) \\
& =o\left(T^{-1}+T^{2}(1-\varphi)^{2}\right)=o(1),
\end{aligned}
$$

$$
\begin{aligned}
& \frac{\sum_{f=2}^{T / 2]} \sum_{g=f+[T / 2]}^{T} \sum_{i=1}^{p} \sum_{j=1}^{p} \boldsymbol{\Sigma}_{\mathbf{i i}} \boldsymbol{\Sigma}_{\mathbf{j} \mathbf{j}} \sum_{h_{1}=-\infty}^{\infty} \sum_{h_{2}=-\infty}^{\infty} \breve{b}_{f, h_{1}} \breve{b}_{g, h_{1}} \breve{b}_{f, h_{2}} \breve{b}_{g, h_{2}}}{\left(T-\frac{3}{2}[T / 2]\right)([T / 2]-1)} \\
& =\frac{\operatorname{tr}(\boldsymbol{\Sigma})^{2}}{\left(T-\frac{3}{2}[T / 2]\right)([T / 2]-1)} \sum_{f=2}^{[T / 2]} \sum_{g=f+[T / 2]}^{T}\left(a_{g-f}^{2}+O\left(a_{g-f}(1-\varphi)\right)+O\left((1-\varphi)^{2}\right)\right) \\
& =O\left(p^{2} T^{-3}+p^{2}(1-\varphi)^{2}\right)=o\left(p^{1 / 2}+p T^{2}(1-\varphi)^{2}\right)=o(p),
\end{aligned}
$$


and

$$
\begin{aligned}
& \frac{\sum_{f=2}^{[T / 2]} \sum_{g=f+[T / 2]}^{T} \sum_{i=1}^{p} \boldsymbol{\Sigma}_{\mathbf{i i}}^{2} \sum_{h=-\infty}^{\infty} \breve{b}_{f, h}^{2} \breve{b}_{g, h}^{2}}{\left(T-\frac{3}{2}[T / 2]\right)([T / 2]-1)} \\
& \leq \frac{\sum_{i=1}^{p} \boldsymbol{\Sigma}_{\mathbf{i i}}{ }^{2}}{\left(T-\frac{3}{2}[T / 2]\right)([T / 2]-1)} \sum_{f=2}^{[T / 2]} \sum_{g=f+[T / 2]}^{T}\left(\sum_{h=-\infty}^{f} \breve{b}_{f, h}^{2}\right)\left(\sum_{h=-\infty}^{f} \breve{b}_{g, h}^{2}\right) \\
& =o\left(p T^{-1}\right)=o\left(p^{1 / 2}\right) \text {. }
\end{aligned}
$$

These ensure that

$$
E S_{\sigma^{2}, 0, l}=a_{0}^{2} \operatorname{tr}\left(\boldsymbol{\Sigma}^{2}\right)+o(p) .
$$

One can similarly obtain the order of $\operatorname{Var}\left(S_{\sigma^{2}, 0, l}\right)$ as follows

$$
\operatorname{Var}\left(S_{\sigma^{2}, 0, l}\right)=o\left(p^{3} T^{-2}+p\right)=o\left(p^{2}\right) .
$$

From (C.40), (C.48) and (C.49), we conclude that

$$
\frac{S_{\sigma^{2}, 0}}{p}=a_{0}^{2} \frac{\operatorname{tr}\left(\boldsymbol{\Sigma}^{2}\right)}{p}+o_{p}(1)
$$

and

$$
\frac{\grave{S}_{\sigma^{2}, 0}}{p}=a_{0}^{2} \frac{\operatorname{tr}\left(\boldsymbol{\Sigma}^{\mathbf{1} / \mathbf{2}}(\mathbf{I}-\mathbf{H}) \boldsymbol{\Sigma}(\mathbf{I}-\mathbf{H}) \boldsymbol{\Sigma}^{\mathbf{1 / 2}}\right)}{p}+o_{p}(1)
$$

This, together with Lemma 2, implies that

$$
\grave{S}_{\sigma^{2}, m}=\frac{\left|\grave{\mu}_{m}\right| \sqrt{2 \frac{\grave{S}_{\sigma^{2}, 0}}{p}}}{\sum_{i=2}^{T} \frac{\grave{x}_{i, i}}{p(T-1)}}=\left(a_{0}+2 \sum_{i=1}^{\infty} a_{i}\right) \sqrt{\frac{2 \operatorname{tr}\left(\boldsymbol{\Sigma}^{\mathbf{1} / \mathbf{2}}(\mathbf{I}-\mathbf{H}) \mathbf{\Sigma}(\mathbf{I}-\mathbf{H}) \boldsymbol{\Sigma}^{\mathbf{1} / \mathbf{2}}\right)}{p}}+o_{p}(1) .
$$

